\title{
Optimal Base-Station Locations in Two-Tiered Wireless Sensor Networks
}

\author{
Jianping Pan, Member, IEEE, Lin Cai, Student Member, IEEE, Y. Thomas Hou, Senior Member, IEEE, \\ Yi Shi, Student Member, IEEE, and Sherman X. Shen, Senior Member, IEEE
}

\begin{abstract}
We consider generic two-tiered Wireless Sensor Networks (WSNs) consisting of sensor clusters deployed around strategic locations, and base-stations (BSs) whose locations are relatively flexible. Within a sensor cluster, there are many small sensor nodes (SNs) that capture, encode, and transmit relevant information from a designated area, and there is at least one application node (AN) that receives raw data from these SNs, creates a comprehensive local-view, and forwards the composite bit-stream toward a BS. This paper focuses on the topology control process for ANs and BSs, which constitute the upper tier of two-tiered WSNs. Since heterogeneous ANs are battery-powered and energy-constrained, their node lifetime directly affects the network lifetime of WSNs. By proposing algorithmic approaches to locate BSs optimally, we can maximize the topological network lifetime of WSNs deterministically, even when the initial energy provisioning for ANs is no longer always proportional to their average bit-stream rate. The obtained optimal BS locations are under different lifetime definitions according to the mission criticality of WSNs. By studying intrinsic properties of WSNs, we establish the upper and lower bounds of maximal topological lifetime, which enable a quick assessment of energy provisioning feasibility and topology control necessity. Numerical results are given to demonstrate the efficacy and optimality of the proposed topology control approaches designed for maximizing network lifetime of WSNs.
\end{abstract}

Index Terms-Network topology, sensor networks, wireless communications, algorithm design, geometric algorithms.

\section{INTRODUCTION}

W IRELESS Sensor Networks (WSNs) and wireless ad hoc networks [2], [3] have attracted intensive research attention in recent years. In addition to many traditional applications enabled by their wired counterparts such as real-time monitoring, search and rescue, emergence response, and field surveys, WSNs have some unparalleled features: quick on-demand (re)deployment and virtually free of coverage constraints. Attracted by their potentials and features, there are extensive research efforts in energyconscious media access control (MAC) [4], [5], [6], [7], variable topology multihop routing [8], [9], [10], localized flow and error control [11], [12], domain-specific application design [13], etc. Although regular computer networks may have similar concerns, the distinct characteristics (e.g., severe constraints of on-board computation, communication, storage, and energy provisioning) of WSNs and the like demand a revisit of the design in traditional network architectures, services, and protocols. As being expected, these multilayer efforts are paving the road to a more efficient WSN deployment in the near future.

We consider two-tiered WSNs, where small sensor nodes (SNs) are deployed in clusters around strategic locations to capture information in terms of video or audio streams, temperature readings, motion measures, and so on. There is

- J. Pan, L. Cai, and S.X. Shen are with the Department of Electrical and Computer Engineering, Centre for Wireless Communications, University of Waterloo, Waterloo, ON N2L 3G1, Canada.

E-mail: \{jpan,cai,xshen\}@bbcr.uwaterloo.ca.

- Y.T. Hou and Y. Shi are with the Bradley Department of Electrical and Computer Engineering, Virginia Tech, Blacksburg, VA 24061.

E-mail: \{thou,yshi\}@vt.edu.

Manuscript received 8 Oct. 2003; revised 7 Apr. 2004; accepted 25 Apr. 2004; published online 27 July 2005.

For information on obtaining reprints of this article, please send e-mail to: tmc@computer.org, and reference IEEECS Log Number TMC-0165-1003. at least one application node (AN) in each cluster; the AN is responsible for receiving raw data sent from SNs, creating a local-view by exploring application-specific correlations among these data and forwarding the composite bit-stream toward a base-station (BS). The BS, which may be relatively distant from ANs when compared with the short SN-AN distance within a cluster, has much better processing and storage capabilities to further increase the value of localviews (e.g., by creating a comprehensive global-view for the entire WSN). The BS also serves as a gateway for WSNs to exchange data and control information with other networks.

Since SNs and ANs are normally battery-powered, it is unlikely, if not impossible, to recharge them economically once they have been deployed in field. If a single $\mathrm{SN}$ runs out of energy, its AN may still have the capability to reconstruct a comprehensive local-view by data sent from other correlated SNs. However, if an AN runs out of energy, the whole cluster coverage is completely lost, which can jeopardize the entire mission in some cases. Therefore, the energy constraint of ANs is the foremost concern for twotiered WSNs. Although ANs can have better energy provisioning than SNs, they also consume energy at a considerably higher rate, due to the transmission of bitstreams over much greater distances. On the other hand, BSs can have extra rechargeable power supplies to meet additional computation, communication, and storage needs. In addition, once being sparsely deployed around strategic locations, SN/AN clusters are stationary or with very low mobility. However, we may have the flexibility to locate recyclable BSs intelligently and to arrange communication activities among ANs and BSs properly. The location and communication arrangement can be determined before WSN initialization, or can be adjusted throughout a mission. Last, but not least, the practical value, or utility, of a WSN heavily depends on the time 


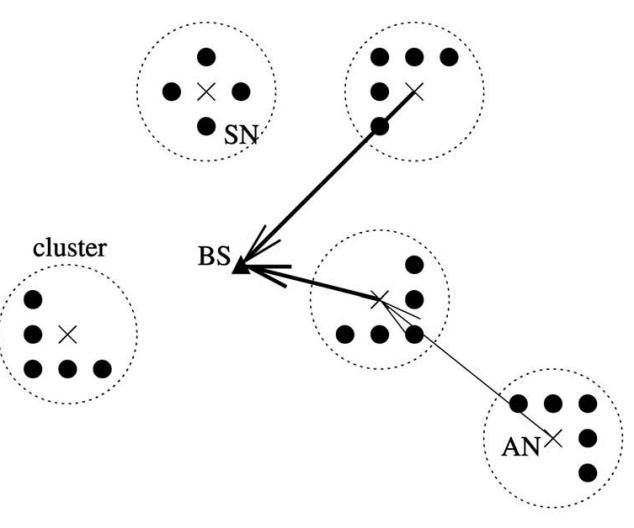

(a)

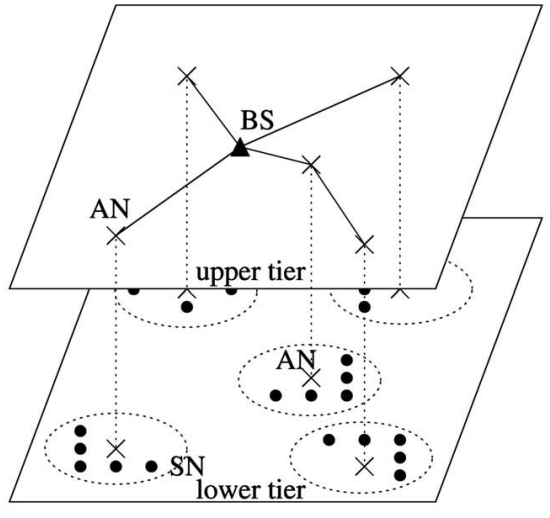

(b)

Fig. 1. A two-tiered architecture of wireless sensor networks. (a) Physical view. (b) Logical view.

duration from network initialization to a point when the WSN fails to maintain enough ANs alive to construct and feed live local-views. Here, we are particularly interested in the lifetime of the entire WSN, which has to be collectively determined by the lifetime of certain ANs. Many factors are involved in determining node lifetime: An energy-conscious MAC can avoid the energy wasted by consistent channel sensing and frequent transmission collisions; an energy-aware routing can balance power consumption among ANs and route around dead or dying ANs; an energy-favored flow or error control scheme can have an asymmetric design with most of its protocol overhead at BSs that are not energy constrained. Non-communicationrelated activities such as node operating and view composition also affect AN lifetime.

We focus on the impact of distance-dominated and communication-related power consumption in WSNs. In particular, we are interested in topology control, an underlying control process even below the traditional ISO/OSI protocol reference model. Given a geographical coverage and a set of information sources, topology control determines where to place SNs, ANs, and BSs, how to allocate initial energy, and how to arrange communications among ANs and BSs, in order to accomplish a mission effectively and efficiently. The network lifetime under this process is referred to as topological lifetime. Our goal is to maximize the topological lifetime of a WSN with regard to a given mission and a certain amount of initial energy by locating BSs optimally with already-located homogeneous or heterogeneous ANs. Once BSs are located, communication activities among ANs and BSs can be further optimized.

The main contribution of this paper is to algorithmically obtain optimal BS locations, under different lifetime definitions according to mission criticality, for WSNs without relying on inter-AN relaying. Our preliminary work in [1] has obtained the optimal BS locations for homogeneous WSNs by assuming that the initial energy of ANs is always proportional to their average bit-stream rate (i.e., bit-rate). Here, we investigate a more generic problem, i.e., how to obtain optimal BS locations for WSNs of heterogeneous ANs. The solution to this problem is of ultimate importance since the initial energy of ANs may not always be proportional to their average bit-rate in practice, especially when node redeployment and energy reprovisioning scenarios are considered. By introducing the novel concept of virtually-stacked-planes, we derive optimal BS locations for heterogeneous WSNs without any energy/bit-rate constraints. We also study intrinsic properties of WSNs, and develop the upper and lower bounds of maximal topological lifetime to enable a quick assessment of energy provisioning feasibility and topology control necessity, which can eliminate unnecessary computation overhead when the optimal BS locations are either trivial, or infeasible to achieve the desired network lifetime with the given resource provisioning. Different from other related work attempting to minimize power consumption for an individual node or along a forwarding path, our work focuses on maximizing the lifetime of the entire WSNs.

The remainder of this paper is organized as follows: In Section 2, we present our system architecture of two-tiered WSNs, their AN power consumption and energy dissipation models, and three definitions of topological network lifetime. In Section 3, by extending an algorithm in Computational Geometry, we develop a family of approaches to optimally locate BSs for both homogeneous and heterogeneous ANs. In addition, we discover some intrinsic properties of WSNs, and derive the upper and lower bounds of maximal topological lifetime to enable a quick feasibility and necessity assessment. Locating BS without relying on inter-AN relaying provides a hard bottom-line when relaying is application-undesirable, energy-unfavorable, physically infeasible, or simply failed in operation. Numerical results are given to demonstrate the efficacy and optimality of the proposed approaches. Further discussions are presented in Section 4. We review related work in Section 5. Section 6 concludes this paper.

\section{System Model}

\subsection{Two-Tiered Wireless Sensor Networks}

A two-tiered WSN, as shown in Fig. 1a, consists of a number of SN/AN clusters and at least one BS. In each cluster, there are many SNs and at least one AN. SNs are responsible for all sensing-related activities. Once triggered by an internal timer or an external event, an SN starts to 
TABLE 1

Symbols

\begin{tabular}{c|l||c|l}
\hline symbol & \multicolumn{1}{|c||}{ description } & symbol & \multicolumn{1}{|c}{ description } \\
\hline \hline$V_{N}$ & a set of $N$ ANs of a WSN & $v_{i}$ & an AN at $\left(x_{i}, y_{i}\right)$ on a plane \\
$b$ & BS (for notation convenience, $\left.v_{0}=b\right)$ & $d_{i}$ & Euclid distance from $v_{i}$ to $b$ \\
$d_{j, k}$ & Euclid distance from $v_{j}$ to $v_{k}$ & $D$ & diameter of $V_{N}, i . e ., D=\max \left\{d_{j, k}\right\}$ \\
$r_{i}(t)$ & data rate generated by $v_{i}$ at time $t$ & $r_{i}$ & when $r_{i}(t)$ is time-invariant \\
$p_{i}(t)$ & power consumption of $v_{i}$ at time $t$ & $p_{i}$ & when $p_{i}(t)$ is time-invariant \\
$e_{i}(t)$ & remaining energy for $v_{i}$ at time $t$ & $e_{i}(0)$ & initial energy allocation for $v_{i}$ \\
$l_{i}$ & node lifetime of $v_{i}$ & $L$ & network lifetime of $V_{N}$ \\
$V_{C}$ & critical node set & $V_{S}$ & supporting node set \\
$C$ & minimal enclosing circle & $\mathcal{C}$ & minimal enclosing cone \\
\hline
\end{tabular}

capture and encode live information sent directly to an AN in the same cluster. SNs are small, low cost, and disposable, and can be densely deployed within a cluster. SNs do not communicate with other SNs in the same or other clusters, and usually are independently operated. ANs, on the other hand, have much more responsibilities than SNs. First, an $\mathrm{AN}$ receives raw data from all active $\mathrm{SNs}$ in the same cluster. It may also instruct SNs to be in sleep, idle, or active state, if some SNs are found to always generate uninterested or duplicated data, thereby allowing these SNs to be reactivated later when some existing active SNs run out of energy. Second, the AN creates an application-specific localview for the whole cluster by exploring correlations among the data sent from SNs. Excessive redundancy in raw data can be alleviated, and the fidelity of captured information should be enhanced. Third, the AN forwards the composite bit-stream toward a BS that generates a comprehensive global-view for the entire WSN. Optionally, ANs can be involved in inter-AN relaying, if such activities are applicable and favorable.

The two-tiered architecture of WSNs is motivated by the latest advances in distributed signal processing and source coding [14], which offer a more flexible balance among reliability, redundancy, and scalability of WSNs. Under this architecture, the primary goal of lower-tier SNs and ANs is to gather data as effectively as possible, while upper-tier ANs and BSs are designed to move information as efficiently as possible. ANs, as shown in Fig. 1b, extracting useful information and composing local-views, are logical bridges for these two tiers. With this functionality partition, we can optimize the performance of individual tiers separately since they are designed for different purposes and have various concerns. Practically, both SNs and ANs are battery-powered. Although ANs can have more initial energy, they also consume energy at a much higher rate due to the transmission of bit-streams to BSs or other ANs that are comparatively far away. Therefore, energy constraints of ANs are our main concern.

Once being deployed, ANs can obtain and report their own location by using on-board GPS (Global Positioning System) receivers, through triangulation with a few refer ence ANs [15], or as instructed by the network operator in a manual deployment. ANs are in sleep state initially, unless they are activated by on-board wake-up circuit. Then, they are instructed with mission schedules, aggregation schemes, and relay routes to cooperatively accomplish a mission with other SN/AN clusters. An SN/AN cluster may undergo a sleep-idle-active cycle repeatedly during its lifetime until the AN exhausts its on-board energy. Once being activated, the AN should feed live local-views or view changes to other ANs and, eventually, to BSs. According to a specific mission, all ANs can be activated at the same time or they can be activated independently. The first style is referred to as synchronized activation and the second one is unsynchronized. An AN can remain in active state once it is activated or it can be in active and inactive (including sleep and idle) states alternatively. The first mode is referred to as continuous activation and the second one is discrete. Although different missions can choose different activation styles and modes, from the viewpoint of remaining energy and topological lifetime, an unsynchronized discrete mission with variable bit-rate ANs can always be converted into an equivalent synchronized continuous mission with constant bit-rate ANs [16], which will be elaborated in Section 2.1.

After ANs have been placed and their locations have been obtained, an immediate challenge is to locate BSs intelligently, so that network lifetime can be maximized. We assume that ANs can communicate with BSs independently, and that BSs are always reachable for ANs as long as ANs can draw enough transmission power from their remaining energy supply. This property, and the characteristics of steady live local-views created by ANs, suggest a deterministic MAC scheme such as TDMA employed by ANs. Although an $\mathrm{SN}$, depending on the amount of sensible information available at a certain moment, can send raw data in burst to its AN, the aggregated live local-views should be relatively smooth and in low volume, whereas the TDMA scheme can save extra control overhead and power consumption encountered by a contention-based MAC scheme. Nevertheless, our study does not rely on any specific MAC schemes since topology control is even under the regular MAC layer. When an imperfect MAC scheme is employed, additional power consumption should be taken into account, and the overall network lifetime is accordingly reduced. In Table 1, we list some symbols used in this paper.

\subsection{Power and Energy Models}

Communication is found to be a dominant source of power consumption in WSNs where live local-views are transmitted over the air. Thus, we focus on communication-related activities for battery-powered ANs since BSs are not energy constrained. For an AN to transmit a 


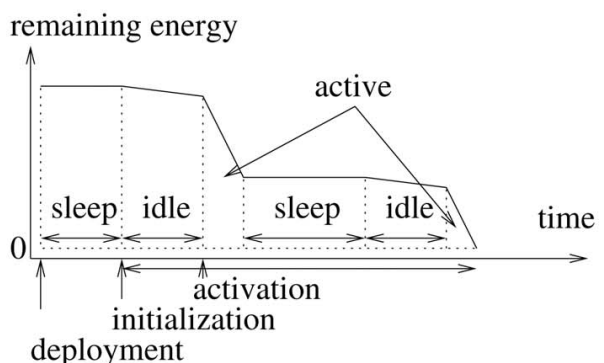

(a)

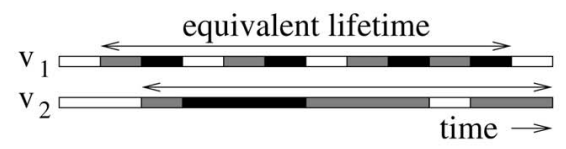

(c)

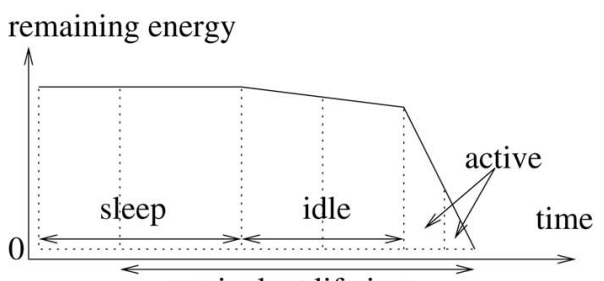

equivalent lifetime

(b)

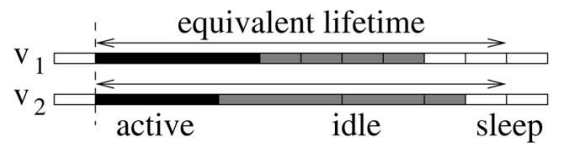

(d)

Fig. 2. Convertibility in activation styles and modes. (a) Discrete activation. (b) Continuous activation. (c) Unsynchronized activation. (d) Synchronized activation.

composite bit-stream at rate $r$ over Euclid distance $d$, the minimal transmission power consumption $p_{t}$ is

$$
p_{t}(r, d)=r\left(\alpha_{1}+\alpha_{2} d^{n}\right),
$$

where $\alpha_{1}$ is a distance-independent term (e.g., the power consumed in transmitter circuit) and $\alpha_{2}$ is a distancedependent term. Equation (1) mainly considers the path loss of exponent $n$, and usually $2 \leq n \leq 4$ for free-space and short-to-medium-range radio communications. Even with a more sophisticated model (e.g., including the power consumed to combat multipath fading and geographical shadowing effects), we can still isolate the distancedominated power consumption empirically. Since the distance-independent power consumption is generally irrelevant to BS locations, it is sufficient to mainly focus on the distance-dependent power consumption when we try to obtain optimal BS locations.

Assume that the initial energy allocation for an $\mathrm{AN}$ is $e(0)$. With a linear energy dissipation model, the node lifetime $l$ of this AN is defined by

$$
\int_{t=t_{0}}^{t_{0}+l} p(t) d t=e(0),
$$

where $t_{0}$ is the time instance when the AN is initialized. Even with a nonlinearity model for conventional batteries (i.e., battery lifetime is determined by both battery capacity and discharge current raised to the Peuker constant), we can still derive $l$ from $e(0)$ and $p(t)$ empirically.

From the viewpoint of remaining energy, as shown in Fig. 2, an unsynchronized discrete mission can always be transformed into an equivalent synchronized continuous mission [16], if the synchronization cost is negligible when compared with the overall communication cost. For instance, Fig. 2a represents a discrete mission. If we group all of sleep, idle, and active states together, we have Fig. $2 b$, which is a continuous mission equivalent in remaining energy. In Fig. 2c, two ANs, $v_{1}$ and $v_{2}$, have unsynchronized activation cycles. However, we can always rearrange the transformed continuous missions to guarantee that they are synchronized at least once. The convertibility is due to the additive property of consumed energy, which is the integral of power consumption over time according to (2). In the rest of this paper, we mainly focus on a synchronized continuous mission with $t_{0}=0$, and the results can be extended to any general missions with arbitrary activation styles and modes.

\subsection{Topological Lifetime Definitions}

For a WSN of $N$ ANs on a plane

$$
\left(V_{N}=\left\{v_{i}=\left(x_{i}, y_{i}\right) \mid i=1 . . N\right\}\right),
$$

given the initial energy allocation $e_{i}(0)$ for $v_{i}$ that generates a composite bit-stream at rate $r_{i}(t)>0$, the node lifetime is $l_{i}$. For topology control, we are interested in network lifetime $(L)$ from network initialization to a point when the WSN cannot maintain enough ANs alive to continue the given mission; the goal of topology control is to maximize topological network lifetime with regard to a certain amount of initial energy provisioning.

According to the criticality of a mission, there are three definitions of topological lifetime:

- $\quad N$-of-N lifetime $L_{N}$ : The mission fails when any ANs run out of energy, i.e., $L_{N}=\min \left\{l_{i}\right\}$ for $1 \leq i \leq N$. The first ANs that run out of energy are denoted as critical ANs in $V_{C}$.

- $\quad K$-of-N lifetime $L_{N}^{K}$ : The mission survives as long as there are at least $K$ ANs alive $(1 \leq K \leq N)$, or the mission fails when $N-K+1$ ANs run out of energy, i.e., $L_{N}^{K}=\min _{N-K+1}\left\{l_{i}\right\}$.

- $\quad m$-in-K-of-N lifetime ${ }^{m} L_{N}^{K}$ : The mission survives as long as all $m$ supporting ANs in $V_{S}$ are alive and overall at least $K$ ANs are alive $(1 \leq m \leq K \leq N)$. In other words, the mission fails if any ANs in $V_{S}$ or $N-K+1$ ANs in $V_{N} \backslash V_{S}$ run out of energy, i.e., ${ }^{m} L_{N}^{K}=\min \left\{L_{m}\left|V_{S}, L_{N-m}^{K-m}\right| V_{N} \backslash V_{S}\right\}$.

Maximizing topological lifetime $L_{N}$ is equivalent to maximizing $\min \left\{l_{i}\right\}$ for $1 \leq i \leq N$, where $\min \left\{l_{i}\right\}$ is the lifetime of critical ANs. For $L_{N}$, it implies that every AN is vital and cannot be substituted by others; while for $L_{N}^{K}$, 


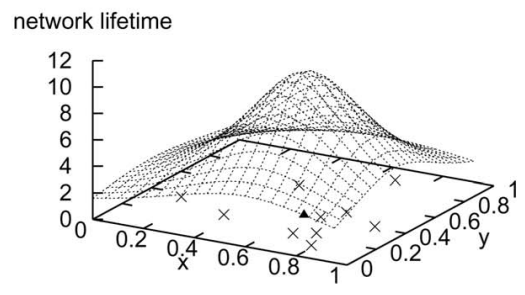

(a)

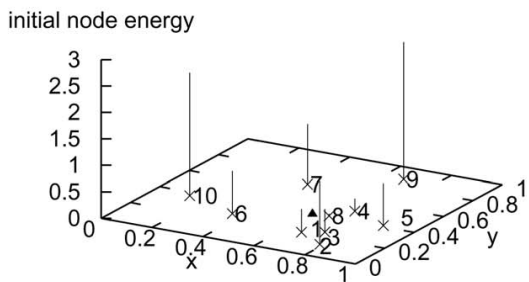

(b)

Fig. 3. Flexible initial energy allocation. (a) Normalized network lifetime. (b) Initial node energy.

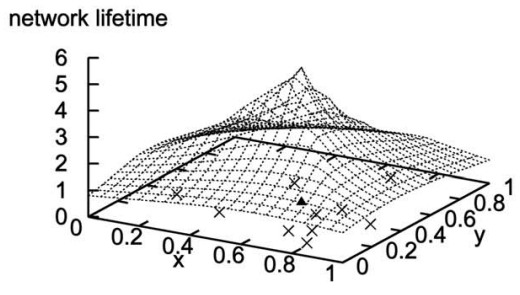

(a)

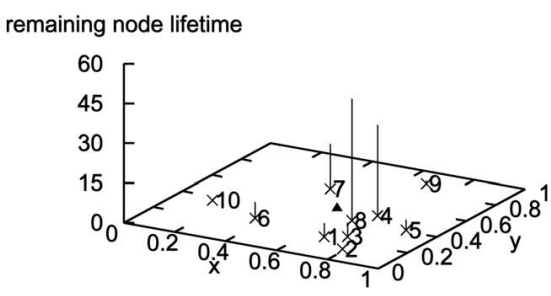

(b)

Fig. 4. Fixed initial energy allocation. (a) Normalized network lifetime. (b) Remaining node lifetime.

there is a certain amount of redundancy among ANs. Under $L_{N}^{K}$, even if some ANs fail, their responsibilities can be taken by nearby ANs (e.g., through cluster reformation), so that the WSN still has the capability to carry on its mission. Normally, $K$ is close to $N$; otherwise, the deployment of ANs has too much redundancy. Obviously, $L_{N} \leq L_{N}^{K}$ when $K<N$; when $K=N, L_{N}^{K}$ degenerates to $L_{N}$. Less obviously, $L_{N}^{K}$ is not the time when the $(N-K+1)$ th AN runs out of energy under the $L_{N}$ definition, which will be explicitly clarified in Section 3.4. The prespecified $m$ supporting ANs are vital to the entire mission; they also play an important role when determining network lifetime ${ }^{m} L_{N}^{K}$. Since $m \leq K \leq N$, we have $L_{N} \leq{ }^{m} L_{N}^{K} \leq L_{N}^{K}$; when $m=0,{ }^{m} L_{N}^{K}$ degenerates to $L_{N}^{K}$.

\section{Optimal Base-Station locations}

We begin with locating a BS $b$ (or $v_{0}=\left(x_{0}, y_{0}\right)$ for notation convenience) for $V_{N}$. We do not rely on inter-AN relaying for reasons such as relaying may be undesirable for applications, relaying may be energy inefficient (e.g., the transmission power saving due to a smaller $d$ in (1) may not compensate the extra overhead in data reception and processing from the standpoint of the entire WSN), or is infeasible due to the limitations in MAC and AN designs (e.g., a simplex or half-duplex MAC with a very limited onboard buffer) or trust concerns (e.g., BSs cannot accept data from an AN that does not generate them). More importantly, the BS location obtained under this context gives a hard bottom-line lifetime even if inter-AN relaying fails due to various reasons in operation.

\subsection{Initial Energy Allocation}

Before obtaining the optimal BS location, we first find that the initial energy allocation $\left\{e_{i}(0) \mid i=1 . . N\right\}$ plays an important role in topology control for WSNs. Fig. 3a gives the normalized lifetime $L_{N}$ with a random BS location for a sample WSN of $N=10$ ANs scattered on a unit square (identified by numbered crosses). A filled triangle on the plane denotes the BS location when network lifetime is maximized through a numerical grid search. Here, we assume that the initial on-board energy can be proportionally allocated for an AN according to its actual power consumption, and that the total energy provisioning for the entire WSN is $N$ units. Let $n=2$ for an easy geometrical illustration. Assume that only the distance-dominated power consumption is considered, i.e., $\alpha_{1}=0$. Therefore, $l_{i}=\frac{e_{i}(0)}{r \alpha_{2} d_{i}^{2}}$, where

$$
r=\frac{\int_{0}^{l} r(t) d t}{l}
$$

according to the convertibility illustrated in Fig. 2. Network lifetime shown in Fig. 3a is normalized with regard to $\frac{1}{r \alpha_{2}}$. Fig. 3b plots the initial AN energy allocation corresponding to the best BS location found in Fig. 3a. It can be seen that the farther away an AN is from the BS, the more initial energy the AN should be granted, so that all ANs can run out of energy at the exactly same time, and no initially provisioned on-board energy will be left unused (i.e., wasted).

However, in practice, we rarely have such flexibility and granularity to allocate initial energy. Homogeneous off-shelf ANs may have different models, but their initial on-board energy (e.g., the number of batteries) is discrete and may only be proportional to the bit-rate at which they generate, i.e., $E(0)=\frac{e_{i}(0)}{r_{i} \alpha_{2}}$ for all ANs and $l_{i}=\frac{E(0)}{d_{i}^{2}}$. In Fig. 4 a, node lifetime is normalized with regard to $E(0)$, which is compatible with that in Fig. 3a. Fig. 4a plots the network lifetime under a fixed allocation scheme for the same sample WSN. As listed in Table 2, with the fixed scheme, the maximal topological lifetime through numerical search drops dramatically from 10.794 to 5.083 unit time. Even with the best BS location, when the entire 
TABLE 2

Statistics of Flexible and Fixed Initial Energy Allocation Schemes

\begin{tabular}{c||ccccc}
\hline$L_{N}$ & min & mean & median & $\max$ & $25 \%-75 \%$ \\
\hline \hline flexible & 1.337 & 4.520 & 3.942 & 10.794 & $2.722-5.800$ \\
fixed & 0.722 & 1.789 & 1.546 & 5.083 & $1.250-2.123$ \\
\hline
\end{tabular}

WSN fails to continue its mission, many ANs still have considerable energy left (but wasted) to keep themselves alive for a while (e.g., 45.831 unit remaining lifetime for $v_{8}$, as shown in Fig. $4 \mathrm{~b}$ ). Table 2 indicates that topology control is crucial for WSNs because, regardless of energy allocation schemes, a randomly-located BS is unlikely to achieve maximal network lifetime. Since the fixed allocation scheme is commonly employed, we adopt it for the rest of this paper.

\subsection{N-of-N Lifetime-Homogeneous ANs}

Although exhaustive searches such as those used in Fig. 3 and Fig. 4 can find the best BS location approximating the optimal one, this approach becomes prohibitively expensive when we have a large coverage and require fine precision. In this section, we will develop an algorithmic approach that optimally locates the BS with the least possible complexity. Fig. $4 \mathrm{~b}$ suggests that the optimal BS location is actually determined by a few critical ANs that run out of energy first. Maximizing $\min \left\{l_{i}=\frac{E(0)}{d_{i}^{n}}\right\}$ is equivalent to minimizing

$$
d=\max \left\{d_{i}=\sqrt{\left(x_{i}-x_{0}\right)^{2}+\left(y_{i}-y_{0}\right)^{2}}\right\},
$$

where $\left(x_{0}, y_{0}\right)$ is the BS location; therefore, $L_{N}=\frac{E(0)}{d^{n}}$. Let $E(0)$ be a constant for all homogeneous ANs. In Section 3.3, we will extend the approach developed here to handle heterogeneous ANs. According to the definition of $d$, the optimal $b$ should be located at the center of a minimal enclosing circle $C$ with minimal radius $d$.

\subsubsection{Properties and Bounds}

First, with a naive approach, we show that such a $C$ does exist. As Fig. 5 sketches, we can always find a circle $C_{0}$ large enough to enclose all ANs in $V_{N}$. Then, we keep the center of the circle intact and reduce its radius until there is a smaller circle $C_{1}$ across an AN in $V_{N}$. Next, we move the center of $C_{1}$ toward the AN and keep it being crossed, which effectively reduces the radius of $C_{1}$, until an even smaller circle $C_{2}$ crosses another $\mathrm{AN}$ in $V_{N}$. If these two ANs are codiameter for $C_{2}$, we can no longer shrink $C_{2}$, and $C=C_{2}$. Otherwise, we move the center of $C_{2}$ toward the line determined by these two ANs, and keep them being crossed, which also reduces the radius of $C_{2}$, until either they become codiameter for an even smaller $C_{3}$, or $C_{3}$ crosses the third AN in $V_{N}$. Now, $C=C_{3}$ and, normally, we need at most 3 nodes to determine a circle on a plane (there might be more than 3 ANs crossed by $C_{3}$ eventually when $V_{N}$ degenerates, but it does not affect our results).

Next, we show that $C$ exists uniquely. As shown in Fig. 5b, assume that $C_{1}$ and $C_{2}$ with minimal radius $d$ are centered at $b_{1}$ and $b_{2}$, respectively. Since all ANs are
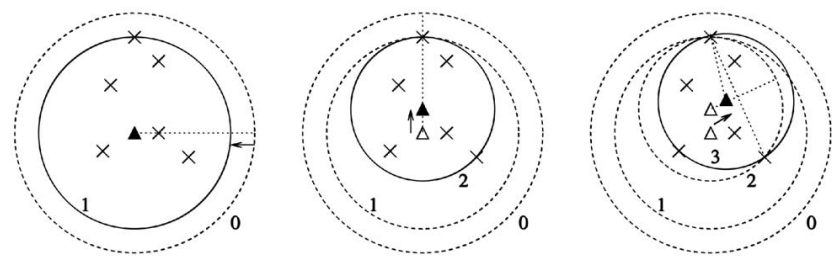

(a)
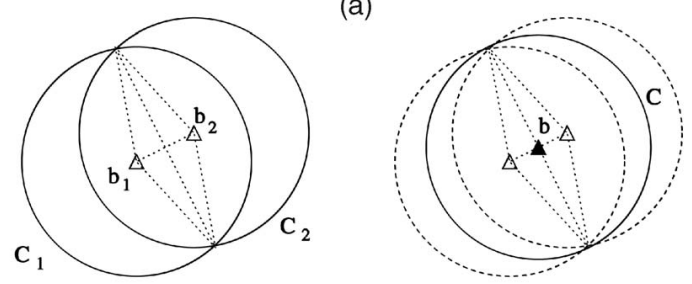

(b)

Fig. 5. Properties of $C$ under the $L_{N}$ definition. (a) Existence. (b) Uniqueness.

enclosed by both $C_{1}$ and $C_{2}$, they should be within an area intersected by $C_{1}$ and $C_{2}$. However, this area can be enclosed by a smaller circle with radius

$$
\sqrt{d^{2}-\left(\frac{d_{b_{1}, b_{2}}}{2}\right)^{2}} \leq d
$$

This fact contradicts the assumption that $C_{1}$ and $C_{2}$ have minimal radius, unless $d_{b_{1}, b_{2}}=0$ or $b_{1}=b_{2}$. The uniqueness of $b$ means that the optimal BS location for a WSN under the $L_{N}$ definition is deterministically unique, so is the $\max L_{N}$.

Finally, as shown in Fig. 6, we derive the upper and lower bounds of $d$. Let AN $E$ and $W$ be the diameter ANs of $V_{N}$, i.e., they are $D$ away. Obviously, $d \geq \frac{D}{2}$; otherwise, $C$ cannot enclose both $E$ and $W$ at the same time. All ANs should be within the area $E-N N-W-S S-E$, where $N N$ and $S S$ are $D$ away from both $E$ and $W$. We find that this area can be enclosed by a circle with radius $\frac{\sqrt{3} D}{2}$ and centered at the middle point of $E$ and $W$. Therefore, $\frac{D}{2} \leq d \leq \frac{\sqrt{3} D}{2}$. With the bounds of $d$, we can obtain the bounds of $\max L_{N}$ and easily assess whether the initial energy allocation is feasible to maintain a WSN for a certain amount of time. If a mission requires its lifetime even greater than the $\max L_{N}$ upper bound, we need to have better energy provisioning, consider inter-AN relaying, or introduce more BSs.

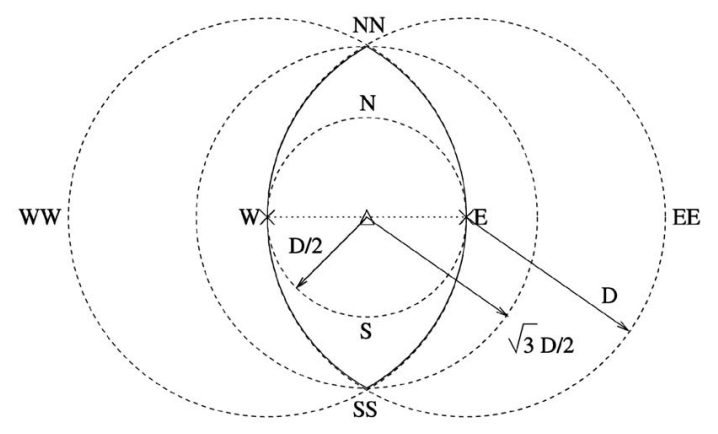

Fig. 6. Upper and lower bounds of $d$. 


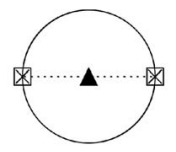

(a)
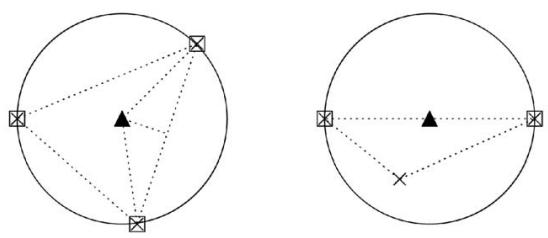

(b)
Fig. 7. $C$ for $V_{N}$ when $N \in\{2,3\}$. (a) $\left|V_{N}\right|=2$. (b) $\left|V_{N}\right|=3$.

\subsubsection{Algorithmic Approach}

The existence property suggests a brute-force approach to find $C$ and $b$. Since $C$ is determined by 2 or 3 ANs in $V_{N}$, there are at most $\left(\begin{array}{l}N \\ 2\end{array}\right)+\left(\begin{array}{l}N \\ 3\end{array}\right)$ such circles. By pairwisely comparing circles enclosing all ANs with regard to their radius, we should find $C$ in finite steps. However, this approach still imposes a computational challenge when $N$ is relatively large.

Therefore, we should propose an algorithmic approach with the least complexity to obtain $C$ deterministically. Fig. 7 gives some examples if $N \in\{2,3\}$. When $N=2$, we have $V_{C}=V_{2}$, and $b$ is at the middle point of these two ANs. When $N=3$, there are two cases:

1. If all 3 angles determined by these $3 \mathrm{ANs}$ are no greater than $\pi / 2, V_{C}=V_{3}$. The largest angle should be no less than $\pi / 3$, and it faces the longest edge no greater than $D$. The view angle from $b$ to the longest edge should be no less than $2 \pi / 3$. With the cosine rule, we have $D \geq \sqrt{3} d$, i.e.,

$$
\frac{D}{2} \leq d \leq \frac{\sqrt{3} D}{3} .
$$

It gives an even tighter upper bound of $d$ to quickly assess the necessity of topology control process and the feasibility of initial energy provisioning since $d$ is in a range less than $\frac{2 \sqrt{3}-3}{6} D \approx 0.077 D$. The derived upper and lower bounds of $\max L_{N}$ are denoted as $L_{N}^{+}$and $L_{N}^{-}$, respectively.

2. If there is 1 (and at most 1 ) angle at AN $v$ greater than $\pi / 2, v$ is noncritical, and $V_{C}=V_{3} \backslash\{v\} .\left|V_{C}\right|=2$, i.e., it degenerates to the case with 2 critical ANs.

Fig. 7 also suggests a recursive approach to obtain $C$. Assume that we have $C_{N-1}$ for $V_{N-1}$, and that its critical set is $V_{C_{N-1}}$. For the Nth AN $v$, if $v$ is enclosed by $C_{N-1}$, then $v \in V_{N} \backslash V_{C_{N}}$ and $C_{N}=C_{N-1}$. Otherwise, $v \in V_{C_{N}}$. As long as we have 2 or 3 critical ANs for any $V_{N}$, we can get $C_{N}$ geometrically. This recursive approach is based on the Welzl algorithm [17] in Computational Geometry, and the main idea is outlined by the Tcl-like pseudocode in Table 3.

$\mathrm{C}\{\mathrm{NC} \mathrm{VC}\}$ is the procedure to find $C$ for noncritical node set $\mathrm{NC}$ and critical node set $\mathrm{VC}$, and it is designed in a divide-and-conquer fashion. If there are 3 nodes in $\mathrm{VC}$, or NC is empty (line 2 in Table 3 ), $C$ can be obtained as shown in Fig. 7 (line 3). Otherwise, randomly pick a $\mathrm{v}$ from $\mathrm{NC}$, and let $\mathrm{NC}^{\prime}=\mathrm{NC} \backslash\{\mathrm{v}\}$, i.e., $\left|\mathrm{NC}^{\prime}\right|=|\mathrm{NC}|-1$. We call $\mathrm{c}\left\{\mathrm{NC}^{\prime} \mathrm{VC}\right\}$ recursively (line 6). After obtaining $\mathrm{C}^{\prime}$ for $\mathrm{NC}^{\prime}$ and $\mathrm{VC}$, we check whether $\mathrm{v}$ is enclosed by $\mathrm{C}^{\prime}$. If so, $\mathrm{C}^{\prime}$ is also applicable for $\mathrm{NC}$ and VC (line 10); otherwise, $\mathrm{V}$ is
TABLE 3

Recursive Algorithm for Determining $C$

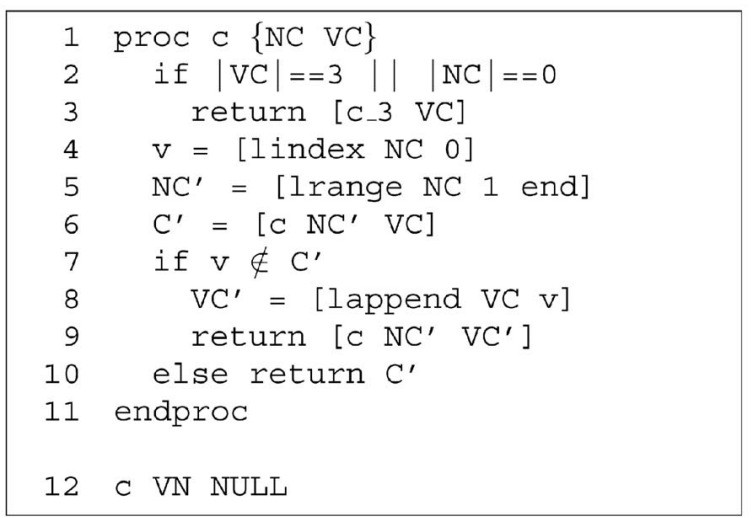

a critical node for $\mathrm{NC}$ and $\mathrm{VC}$. Let $\mathrm{VC}^{\prime}=\mathrm{VC} \cup\{\mathrm{v}\}$, and we call $c\left\{\mathrm{NC}^{\prime} \mathrm{VC}^{\prime}\right\}$ recursively again (line 9). Line 12 in Table 3 gives the initial call on procedure $\mathrm{C}$ when we do not have any knowledge of $\mathrm{VC}$ (i.e., $\mathrm{VC}=\mathrm{NULL}$ ) and treat all nodes as noncritical in NC (i.e., $\mathrm{NC}=\mathrm{VN}$ ).

Since 3 critical nodes of $V_{N}$ are sufficient to determine a $C_{N}$, if we have a perfectly-randomized NC at each step, the probability of line 9 being executed is $p \leq \frac{3}{|\mathrm{NC}|}$ (if $V_{N}$ degenerates, it actually speeds up the algorithm in Table 3, since line 8 and line 9 are less likely to be executed). Assume that the complexity of procedure $\mathrm{c}$ with $i$ known critical nodes and $j$ assumed noncritical nodes is $O_{i, j}$, then

$$
O_{i, j} \leq O_{i, j-1}+O(1)+\frac{3-i}{j} O_{i+1, j-1},
$$

where $O(1)$ is the constant cost (i.e., in lines $2,4,5$, 7, etc.)

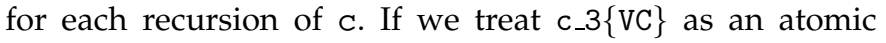
operation, i.e., $O_{3,}=O_{r, 0}=O(1)$, the time complexity of c $\{$ VN NULL $\}$ is $O_{0, n} \sim O(N)$. Actually, this is the best complexity that we can achieve algorithmically [17] since we need to examine every $\mathrm{AN}$ in $V_{N}$ at least once to determine whether the AN is in $V_{C}$ or not, which mandates the least achievable complexity $O(N)$ for any possible algorithms to obtain $C_{N}$.

For the sample WSN, the $C$ and the optimal BS location (filled triangle) obtained through the algorithm described in Table 3 are illustrated in Fig. 8. The critical ANs are additionally denoted with rectangles, and $V_{C}=\left\{v_{2}, v_{9}, v_{10}\right\}$. $\max L_{N}=5.504$ unit time, which is considerably better than the best one (5.083 unit time) obtained through an exhaustive grid search in Fig. 4a. According to $D$, we can obtain the upper and lower bounds of $\max L_{N}, L_{N}^{+}=6.812$, and $L_{N}^{-}=$ 5.110 unit time, respectively. If the desired network lifetime is less than 5.110 unit time, just placing the BS at the middle point of any two diameter ANs can satisfy the mission requirement. If the desired lifetime is more than 6.812 unit time, we even do not need to run the algorithm, since the result will not meet the requirement at all. This quick assessment can speed up topology control by avoiding some unnecessary or doomed computations. 


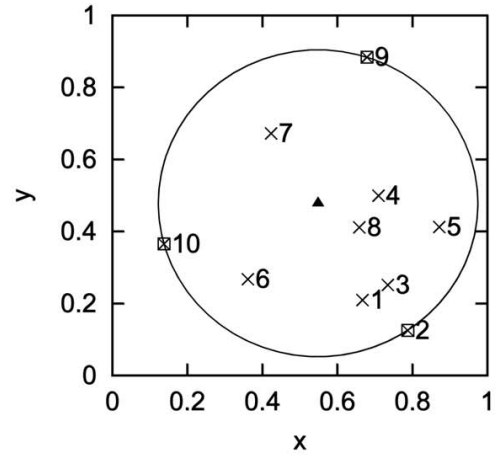

(a)

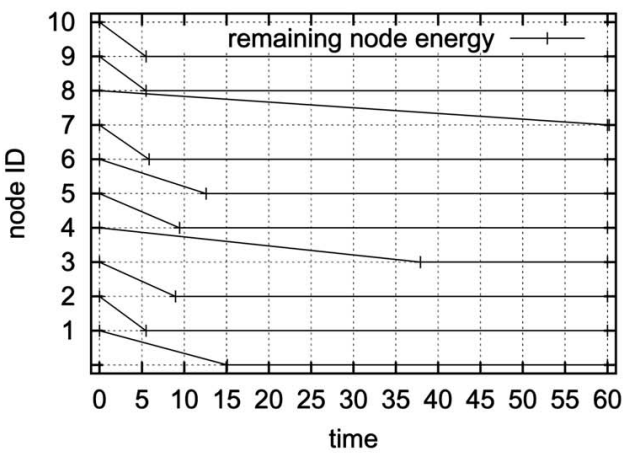

(b)

Fig. 8. $N$-of- $N$ lifetime. (a) Optimal $b$ for $V_{10}$. (b) Node energy and lifetime.

\subsection{N-of-N Lifetime-Heterogeneous ANs}

In Section 3.2, we assumed that $E(0)=\frac{e_{i}(0)}{r_{i} \alpha_{2}}$ is a constant for all ANs. However, in practice, such node homogeneity cannot be always guaranteed. For example, the initial onboard energy of ANs built by different vendors may not be proportional to the bit-rate at which they generate, or the WSN redeployment scenarios are considered (e.g., new ANs join the system long after old ANs have been activated). Therefore, we should develop algorithmic approaches to obtain optimal BS locations for heterogeneous ANs.

According to node lifetime definition $l_{i}=\frac{e_{i}(0)}{r_{i} \alpha_{2} d_{i}^{n}}$, let

$$
k_{i}=\left(\frac{e_{i}(0)}{r_{i} \alpha_{2}}\right)^{\frac{1}{n}}
$$

Maximizing network lifetime $L_{N}$ is equivalent to maximizing $\min \left\{l_{i}=\left(\frac{k_{i}}{d_{i}}\right)^{n}\right\}$. In other words, to locate the BS optimally, $\max \left\{\frac{d_{i}}{k_{i}}\right\}$ should be minimized, where

$$
d_{i}=\sqrt{\left(x_{i}-x_{0}\right)^{2}+\left(y_{i}-y_{0}\right)^{2}},
$$

and $b=\left(x_{0}, y_{0}\right)$ is the BS location.

Without loss of generality, we assume that

$$
k_{1} \leq k_{2} \leq \cdots \leq k_{N} .
$$

When $k_{i}$ s are different, the optimal BS location is determined by not only the two-dimension location $\left(x_{i}, y_{i}\right)$ of ANs, but also $k_{i}$. As shown in Fig. 9, we introduce the concept of stacked planes (i.e., $P_{0}, P_{1}, \cdots$, and $P_{N}$ in a virtual third dimension) to visualize this process. The distance between $P_{0}$ and $P_{i}$ is $k_{i}$. The Cartesian coordinates of the BS are $\left(x_{0}, y_{0}, 0\right)$, and the coordinates of the $i$ th $\mathrm{AN}$ are $\left(x_{i}, y_{i},-k_{i}\right)$, i.e., the $i$ th $\mathrm{AN}$ is on $P_{i}$. Obtaining the optimal BS location $b$ for heterogeneous ANs is equivalent to obtaining a minimal enclosing cone $\mathcal{C}$ which has vertex at $b$ on $P_{0}$ and includes all $v_{i} \in V_{N}$ on $P_{i}$ for $1 \leq i \leq N$. The intersection of $\mathcal{C}$ and $P_{i}$ is a circle $C_{i}$. For radius $R_{i}$ of $C_{i}$, we have $R_{1}: R_{2}: \cdots: R_{N}=k_{1}: k_{2}: \cdots: k_{N}$, i.e., $R=R_{i} / k_{i}$ is a constant and $\max \left\{\frac{d_{i}}{k_{i}}\right\} \leq R$. Obviously, maximizing $L_{N}$ is equivalent to minimizing $R$ of $\mathcal{C}$. Similar to minimal enclosing circle $C$, where homogeneous ANs crossed by $C$ are critical, heterogeneous ANs on the surface of $\mathcal{C}$ are critical, i.e., these ANs run out of energy first by definition.

\subsubsection{Properties}

Similar to the analysis in Section 3.2.1, we can show that such a $\mathcal{C}$ does exist. As shown in Fig. 10a, we can always find a cone $\mathcal{C}_{0}$ large enough to enclose all $v_{i} \in V_{N}$ on $P_{i}$. Then, we keep the vertex of $\mathcal{C}_{0}$ intact, and reduce cone span, until an AN $v_{i}=\left(x_{i}, y_{i},-k_{i}\right)$ on $P_{i}$ is on the cone surface of $\mathcal{C}_{1}$. Next, we move the vertex of $\mathcal{C}_{1}$ toward $\left(x_{i}, y_{i}, 0\right)$ on $P_{0}$, and keep $v_{i}$ on cone surface, which effectively reduces cone span, until another AN $v_{j}=\left(x_{j}, y_{j},-k_{j}\right)$ on $P_{j}$ is on the cone surface of $\mathcal{C}_{2}$. If the vertex of $\mathcal{C}_{2}$ is collinear with $\left(x_{i}, y_{i}, 0\right)$ and $\left(x_{j}, y_{j}, 0\right)$, we can no longer shrink $\mathcal{C}_{2}$; therefore, $\mathcal{C}=\mathcal{C}_{2}$ and $V_{C}=\left\{v_{i}, v_{j}\right\}$. Otherwise, we move the vertex of $\mathcal{C}_{2}$ toward the line determined by $\left(x_{i}, y_{i}, 0\right)$ and $\left(x_{j}, y_{j}, 0\right)$ on $P_{0}$, and keep $v_{i}$ and $v_{j}$ on cone surface, which also reduces cone span, until the vertex of a smaller cone $\mathcal{C}_{3}$ is collinear with $\left(x_{i}, y_{i}, 0\right)$ and $\left(x_{j}, y_{j}, 0\right)$, or the third AN $v_{k}$ is on the cone surface of $\mathcal{C}_{3}$; therefore, $\mathcal{C}=\mathcal{C}_{3}$ and $V_{C}=\left\{v_{i}, v_{j}, v_{k}\right\}$. Normally, we need at most 3 ANs on these virtually-stackedplanes to determine a minimal enclosing cone $\mathcal{C}$ with minimal $R$.

Next, we show that such a $\mathcal{C}$ with minimal $R$ exists uniquely. Assume that there are $\mathcal{C}_{1}$ and $\mathcal{C}_{2}$ of the same minimal $R_{i}$ on $P_{i}$ and with the vertex at $b_{1}=\left(x_{1}, y_{1}, 0\right)$ and $b_{2}=\left(x_{2}, y_{2}, 0\right)$ that are $d$ away. Obviously, $d \leq 2 R_{i}$ for $1 \leq i \leq N$. Similar to the argument in Section 3.2.1, on each plane, there is a smaller circle $C_{i}^{\prime}$ centered at

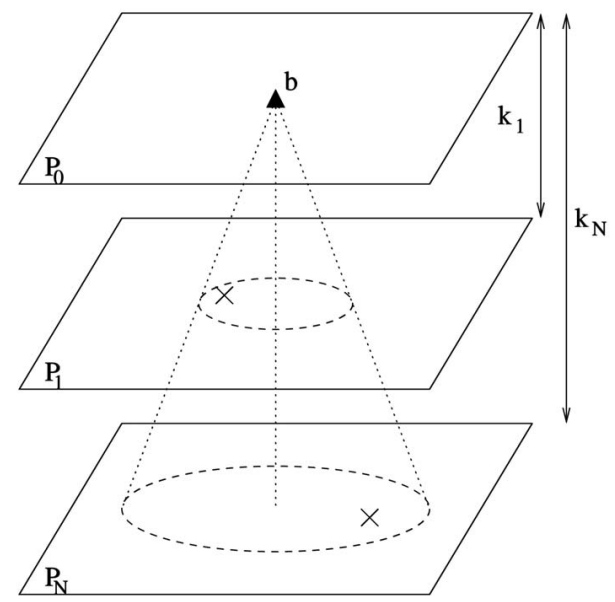

Fig. 9. Heterogeneous ANs on stacked planes. 

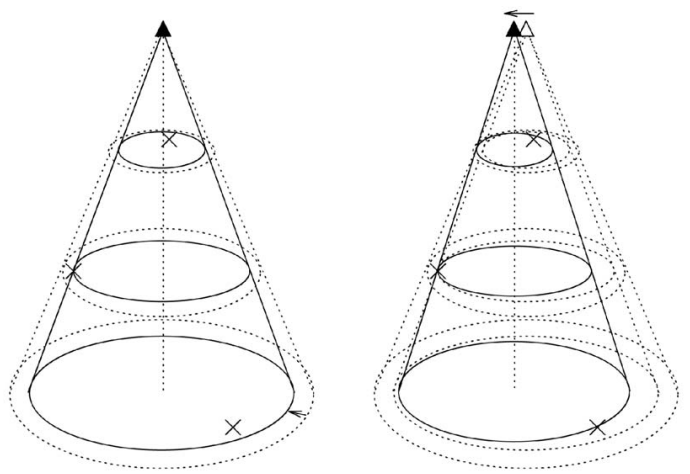

(a)
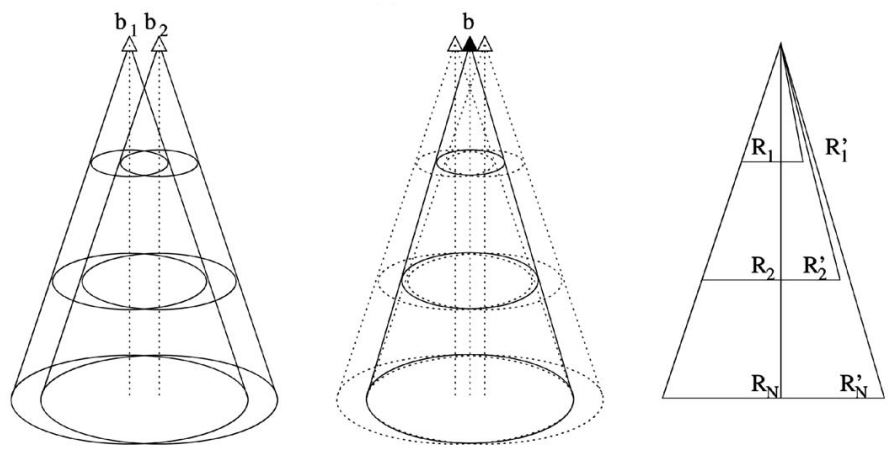

(b)

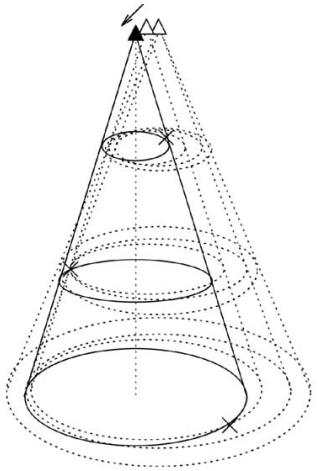

Fig. 10. Properties of $\mathcal{C}$ under the $L_{N}$ definition. (a) Existence. (b) Uniqueness.

$$
\left(\frac{x_{1}+x_{2}}{2}, \frac{y_{1}+y_{2}}{2},-k_{i}\right)
$$

with radius

$$
\sqrt{R_{i}^{2}-\left(\frac{d}{2}\right)^{2}}
$$

enclosing ANs on $P_{i}$. As shown in Fig. 10b, these smaller circles can be enclosed by a smaller cone $\mathcal{C}$ with vertex at $\left(\frac{x_{1}+x_{2}}{2}, \frac{y_{1}+y_{2}}{2}, 0\right)$. To illustrate this, without loss of generality, assume that there are $C_{1}^{\prime}$ and $C_{N}^{\prime}$ on $P_{1}$ and $P_{N}$ with radius

$$
R_{1}^{\prime}=\sqrt{R_{1}^{2}-\left(\frac{d}{2}\right)^{2}}
$$

and $R_{N}^{\prime}=\sqrt{R_{N}^{2}-\left(\frac{d}{2}\right)^{2}}$, respectively. For $R_{1}^{\prime}$ and $R_{N}^{\prime}$, we have

$$
\begin{aligned}
R_{1}^{\prime} & =\sqrt{\left(\frac{R_{N} k_{1}}{k_{N}}\right)^{2}-\left(\frac{d}{2}\right)^{2}}=\frac{k_{1}}{k_{N}} \sqrt{R_{N}^{2}-\left(\frac{d}{2} \frac{k_{N}}{k_{1}}\right)^{2}} \\
& \leq \frac{k_{1}}{k_{N}} \sqrt{R_{N}^{2}-\left(\frac{d}{2}\right)^{2}}=\frac{k_{1}}{k_{N}} R_{N}^{\prime} .
\end{aligned}
$$

The inequity is due to the fact that $k_{1} \leq k_{N}$. Recursively, $R_{i}^{\prime} \leq \frac{k_{i}}{k_{n}} R_{N}^{\prime}$, and there is a $\mathcal{C}$ with a smaller $R^{\prime}$ enclosing all ANs, which contradicts the existence of $\mathcal{C}_{1}$ and $\mathcal{C}_{2}$, unless $b_{1}=b_{2}$ or $\mathcal{C}$ is unique.

\subsubsection{Algorithmic Approach}

To obtain $\mathcal{C}$ algorithmically, we first consider the cases with a small $N \in\{2,3\}$. When $N=2$, as shown in Fig. 11, $b$ is on the line of $v_{1} v_{2}$, and $d_{b, v_{1}}: d_{b, v_{2}}=k_{1}: k_{2}$, i.e., $\max \left\{d_{b, v_{1}} / k_{1}, d_{b, v_{2}} / k_{2}\right\}=d_{b, v_{1}} / k_{1}=d_{b, v_{2}} / k_{2}$. This can be clarified with the following argument:

1. For an arbitrary point $p$ on line $v_{1} v_{2}$, if $p$ is between $b$ and $v_{1}, d_{p, v_{2}}>d_{b, v_{2}}$ and $d_{p, v_{1}}<d_{b, v_{1}}$. Thus,

$$
\begin{aligned}
\max \left\{d_{p, v_{1}} / k_{1}, d_{p, v_{2}} / k_{2}\right\} & =d_{p, v_{2}} / k_{2}>d_{b, v_{2}} / k_{2} \\
& =\max \left\{d_{b, v_{1}} / k_{1}, d_{b, v_{2}} / k_{2}\right\} .
\end{aligned}
$$

Similarly, if $p$ is between $b$ and $v_{2}$,

$$
\begin{aligned}
\max \left\{d_{p, v_{1}} / k_{1}, d_{p, v_{2}} / k_{2}\right\} & =d_{p, v_{1}} / k_{1}>d_{b, v_{1}} / k_{1} \\
& =\max \left\{d_{b, v_{1}} / k_{1}, d_{b, v_{2}} / k_{2}\right\}
\end{aligned}
$$

2. For an arbitrary point $p$ outside line $v_{1} v_{2}$,

$$
d_{p, v_{1}}+d_{p, v_{2}}>d_{b, v_{1}}+d_{b, v_{2}},
$$

i.e., either $d_{p, v_{1}} / k_{1}>d_{b, v_{1}} / k_{1}$ or

$$
d_{p, v_{2}} / k_{2}>d_{b, v_{2}} / k_{2}=d_{b, v_{1}} / k_{1} .
$$

Thus,

$$
\begin{aligned}
& \max \left\{d_{p, v_{1}} / k_{1}, d_{p, v_{2}} / k_{2}\right\}>d_{b, v_{1}} / k_{1}= \\
& \max \left\{d_{b, v_{1}} / k_{1}, d_{b, v_{2}} / k_{2}\right\} .
\end{aligned}
$$




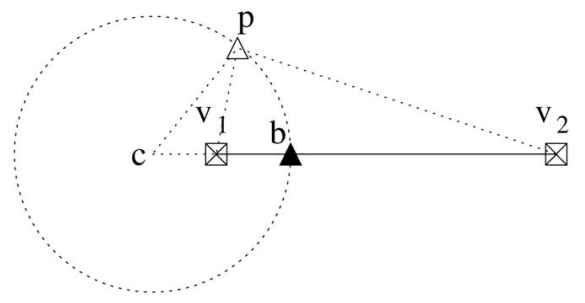

Fig. 11. $\mathcal{C}$ for $V_{N}$ when $N=2$.

TABLE 4

Internode Distance for $V_{N}$ When $N=2$

\begin{tabular}{c||c}
\hline inter-node & distance \\
\hline \hline$v_{1} b$ & $\frac{k_{1}}{k_{2}+k_{1}} d_{v_{1}, v_{2}}$ \\
$v_{2} b$ & $\frac{k_{2}}{k_{2}+k_{1}} d_{v_{1}, v_{2}}$ \\
$c v_{1}$ & $\frac{k_{1}^{2}}{k_{2}^{2}-k_{1}^{2}} d_{v_{1}, v_{2}}$ \\
$p v_{1}$ & $\frac{k_{1}}{k_{2}} p v_{2}$ \\
$p c$ & $\frac{k_{2} k_{1}}{k_{2}^{2}-k_{1}^{2}} d_{v_{1}, v_{2}}$
\end{tabular}

Since subcases 1 and 2 cover all possible locations for $p, b$ should be the optimal BS location for $V_{2}$ and $V_{C}=V_{2}$. As shown in Appendix A.1, all points whose distances to $v_{1}$ and $v_{2}$ satisfy ratio $k_{1}: k_{2}$ form a circle that centers at $c$ and encloses $v_{1}$ when $k_{1}<k_{2}$ (or encloses $v_{2}$ when $k_{1}>k_{2}$ ); therefore, geometrically, $b$ is the intersection of such a circle and line $v_{1} v_{2}$. Table 4 lists distances among $v_{1}, v_{2}, b$, and $p$ whose distances to $v_{1}$ and $v_{2}$ satisfy ratio $k_{1}: k_{2}$. For $v_{1}=$ $\left(x_{1}, y_{1}\right)$ and $v_{2}=\left(x_{2}, y_{2}\right)$,

$$
b=\left(\frac{k_{2} x_{1}+k_{1} x_{2}}{k_{1}+k_{2}}, \frac{k_{2} y_{1}+k_{1} y_{2}}{k_{1}+k_{2}}\right)
$$

and

$$
c=\left(\frac{k_{2}^{2} x_{1}-k_{1}^{2} x_{2}}{k_{2}^{2}-k_{1}^{2}}, \frac{k_{2}^{2} y_{1}-k_{1}^{2} y_{2}}{k_{2}^{2}-k_{1}^{2}}\right),
$$

where $k_{1} \neq k_{2}$.

When $N=3$, similar to Section 3.2.2, we can have 2 possible cases: Either one of $\left\{v_{1}, v_{2}, v_{3}\right\}$ is noncritical, or all $v_{i}$ s for $1 \leq i \leq 3$ are critical. Without loss of generality, in
Fig. 12a, assume that $p_{3}$ is on line $v_{1} v_{2}$ and minimizes $\frac{d_{v_{1} p_{3}}}{k_{1}}=\frac{d_{p_{3} v_{2}}}{k_{2}}$. If $\frac{d_{v_{3} p_{3}}}{k_{3}}<\frac{d_{v_{1} p_{3}}}{k_{1}}=\frac{d_{p_{3} v_{2}}}{k_{2}}$ or

$$
\max \left\{\frac{d_{v_{i} p_{3}}}{k_{i}}, 1 \leq i \leq 3\right\}=\max \left\{\frac{d_{v_{i} p_{3}}}{k_{i}}, 1 \leq i \leq 2\right\},
$$

we have $b=p_{3}$ since $v_{3}$ is noncritical with regard to $k_{3}$ and $b$. According to the case when $N=2$, only $b=p_{3}$ can minimize $\max \left\{\frac{d_{v_{i p}}}{k_{i}}, 1 \leq i \leq 2\right\}$ for any $p$ on the plane.

If all $3 \mathrm{ANs}$ are critical, all points with distances to $v_{2}$ and $v_{3}$ satisfying ratio $k_{2}: k_{3}$ form a circle $C_{1}$; similarly, all points with distances to $v_{1}$ and $v_{3}$ satisfying $k_{1}: k_{3}$ form another circle $C_{2}$. If and only if the BS locates on $C_{1}$, both $v_{2}$ and $v_{3}$ have the same lifetime; similarly, if and only if the BS locates on $C_{2}$, both $v_{1}$ and $v_{3}$ have the same lifetime. Since all 3 ANs are critical (i.e., they have the same lifetime), the optimal BS must locate at the intersection of $C_{1}$ and $C_{2}$. Normally, $C_{1}$ and $C_{2}$ intersect at two points $b_{1}$ and $b_{2}$, respectively, where $\frac{d_{b_{1} v_{1}}}{k_{1}}=\frac{d_{b_{1} v_{2}}}{k_{2}}=\frac{d_{b_{1} v_{3}}}{k_{3}}=R_{1}, \frac{d_{b_{2} v_{1}}}{k_{1}}=\frac{d_{b_{2} v_{2}}}{k_{2}}=\frac{d_{b_{2} v_{3}}}{k_{3}}=R_{2}$, and $R_{1}<R_{2}$. Therefore, $b_{1}$ is the optimal BS location, as illustrated in Fig. 12b. In Appendix A.2, we show that if $V_{C}=V_{3}, b$ should be inside the triangle determined by $V_{3}$, where $C_{1}, C_{2}$, and $C_{3}$ intersect.

Once we obtain $b$ for a small number of heterogeneous ANs geometrically, we can apply the recursive algorithm outlined in Table 3 to optimally locate the BS for any number of heterogeneous ANs. The approach is similar since $\mathcal{C}$ is also determined by a small number of critical ANs. Assume that we have $\mathcal{C}_{N-1}$ for $V_{N-1}$ with critical node set $V_{C_{N-1}}$. For the Nth AN $v$, if $v$ on its stacked plane is enclosed by cone $\mathcal{C}_{N-1}, v$ is noncritical, and $\mathcal{C}_{N}=\mathcal{C}_{N-1}$. Otherwise, $v$ is a critical AN and the recursive algorithm is called again with this additional critical AN. If we treat the procedure to obtain $\mathcal{C}$ when $N \in\{2,3\}$ as an atomic operation, similar to the argument in Section 3.2.2, the complexity of $\mathcal{C}$ for $V_{N}$ is also $O(N)$, which is the least complexity that can be algorithmically achieved.

Since heterogeneous ANs share the same underlying divide-and-conquer principle during topology control as

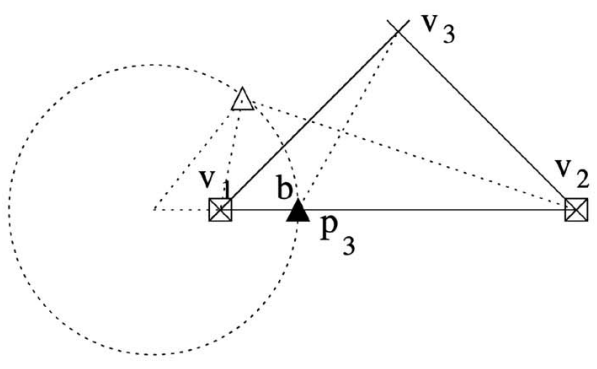

(a)

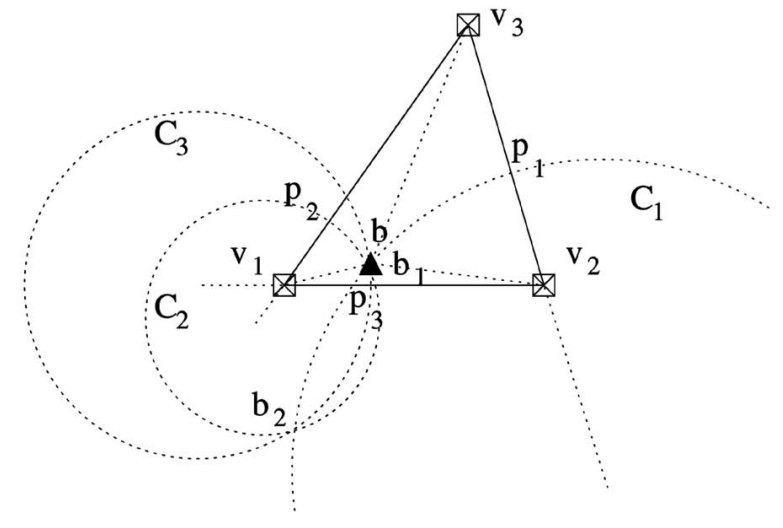

(b)

Fig. 12. $\mathcal{C}$ for $V_{N}$ when $N=3$. (a) $\left|V_{C}\right|=2$. (b) $\left|V_{C}\right|=3$. 


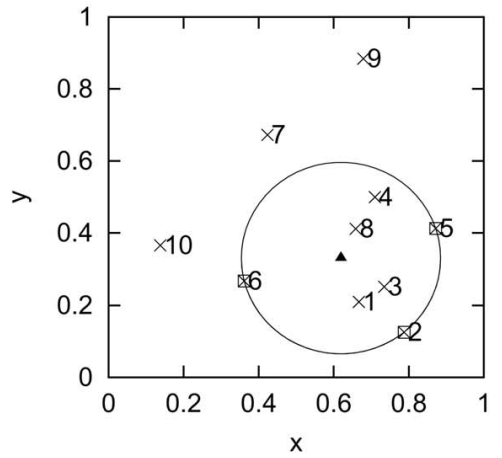

(a)

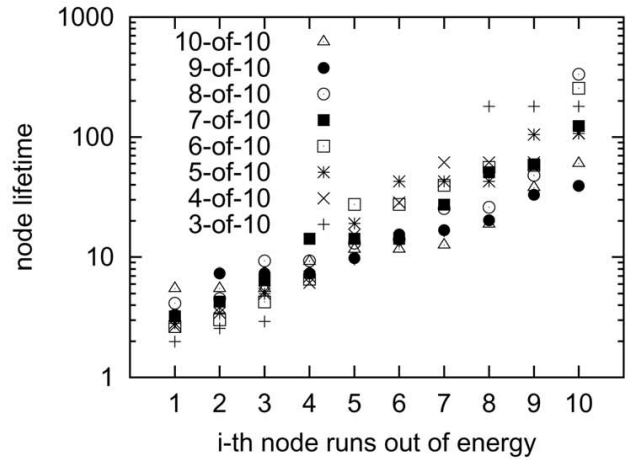

(b)

Fig. 13. $K$-of-N lifetime. (a) Optimal $b$ for $K=7$. (b) $L_{N}^{K}$ when $K=3 . .10$.

homogeneous ANs, in the rest of this paper, we mainly focus on WSNs consisting of homogeneous ANs, and the results can be extended to the cases of heterogeneous ANs accordingly.

\subsection{K-of-N Lifetime}

The optimal BS locations under the $L_{N}^{K}$ lifetime definition should also exist, but the uniqueness property of such optimal locations for a general $K$ cannot be always guaranteed for WSNs adopting $L_{N}^{K}$ lifetime with some easy-to-find counterexamples (e.g., a dumbbell-like WSN coverage).

The existence property also suggests a brutal-force approach to obtain $C_{N}^{K}$, i.e., by choosing any $K$ ANs from $V_{N}$ and executing the developed $L_{K}$ approach repeatedly. Since there are $\left(\begin{array}{l}N \\ K\end{array}\right)$ combinations, by comparing them pairwisely, $\max L_{N}^{K}=\max \left\{L_{K} \mid \forall V_{K} \subset V_{N}\right\}$. However, the definition of $V_{C}$ and the property of $\left|V_{C}\right| \leq 3$ for a general $V_{N}$ can help develop a better algorithmic approach.

When removing an AN $v$ from $V_{N}$, if $v \notin V_{C}$, network lifetime, as well as the optimal BS location, will not change. Only if a $v \in V_{C}$ is removed, the optimal BS location may change accordingly to prolong network lifetime. Since $2 \leq$ $\left|V_{C}\right| \leq 3$ for a general $V_{N}$, we can build a $2-3$ search tree by removing 1 critical $\mathrm{AN}$ at each step (if $V_{N}$ degenerates, removing a redundant critical AN neither prolongs network lifetime nor changes the optimal BS location). The span and depth of this search tree are limited, and we have at most $3^{N-K}$ leaf node-sets to compare. For each node-set of $n$ ANs, the time complexity to locate the BS optimally is $O(n)$. When $N$ is large and $K$ is close to $N$ (i.e., $3^{N-K}<<\left(\begin{array}{l}N \\ K\end{array}\right)$ ), this approach is much more efficient than the brutal-force one. We can further improve the efficiency of this approach, if we have logged the history when building $C_{N}$. Since many node-sets of this search tree have already been examined, we can obtain BS locations and critical AN sets for them directly.

Fig. 13a shows the optimal BS location under $L_{N}^{7}$ for the sample WSN. After ANs $\left\{v_{7}, v_{9}, v_{10}\right\}$ run out of energy, ANs $\left\{v_{2}, v_{5}, v_{6}\right\}$ become critical for the remaining 7 ANs. Fig. 13b further plots $L_{N}^{K}$ s for $K \in\{3,4, \cdots, 10\}$. The vertical axis in $\log$ scale is the time when the $i$ th AN, indexed by the horizontal axis, runs out of energy. For $K=7$, we find that the 4th AN runs out of energy at 14.191 unit time after network initialization, so $\max L_{10}^{7}=14.191$ unit time. Clearly, the smaller $K$, the larger $L_{N}^{K}$, as assessed in Section 2.3. Fig. 13 also validates the proposition that $L_{N}^{K}$ is not the time when the $(N-K+1)$ th AN runs out of energy under the $L_{N}$ lifetime definition. In this sample WSN, the 4th AN under $L_{10}^{10}$ runs out of energy at 9.186 unit time, which is considerably less than $L_{10}^{7}$.

\section{5 m-in-K-of-N Lifetime}

The optimal BS locations for ${ }^{m} L_{N}^{K}$ lifetime should exist, but may not always be unique as well, as ${ }^{m} L_{N}^{K}$ is a special case of $L_{N}^{K}$ by definition. For ${ }^{m} L_{N}^{K}$, once a supporting AN runs out of energy, the entire mission fails immediately. Similar to the possible brutal-force approach for $L_{N}^{K}$, we can find the optimal BS location by choosing $K-m$ ANs from $N-m$ nonsupporting ANs and applying the designed $L_{K}^{K}$ approach repeatedly together with $m$ supporting ANs. There are in total $\left(\begin{array}{c}N-m \\ K-m\end{array}\right)$ combinations of nonsupporting ANs, and $\max ^{m} L_{N}^{K}=\max \left\{L_{K}^{K}\right\}$ by comparing them pairwisely.

By slightly modifying the $L_{N}^{K}$ algorithm, we can obtain an algorithmic approach for ${ }^{m} L_{N}^{K}$. When removing a critical AN from $V_{C}$, if it is also a supporting $\mathrm{AN}$, the spanning phase of the current search tree branch stops. The resultant search tree is no longer always complete. To obtain $\max ^{m} L_{N}^{K}$, we only need to compare the existing leaf node-sets. For each node-set of $n$ ANs, the complexity to locate the BS is still $O(n)$. Under ${ }^{m} L_{N}^{K}$, the algorithm to locate the BS optimally may finish even when there are more than $K$ remaining ANs (as both $V_{C}$ and $V_{S}$ determine network lifetime). Since we only have a partial node-set tree to search, it is clear that $\max ^{m} L_{N}^{K} \leq \max L_{N}^{K}$.

In summary, we have developed a family of approaches to obtain the optimal BS locations (under the N-of-N, K-of-N, and $m$-in-K-of- $N$ network lifetime definitions) for both homogeneous and heterogeneous ANs. The developed algorithmic approaches share the same divide-and-conquer principle due to the intrinsic relationship among these lifetime definitions. With these approaches, we can maximize network lifetime with regard to a given amount of 


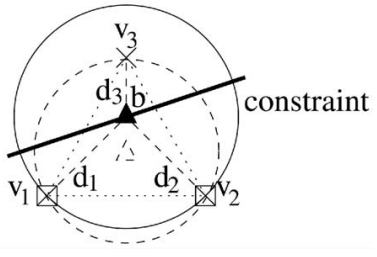

Fig. 14. $V_{3}$ with a linear constraint.

initial energy provisioning. Moreover, the $L_{N}$ approach is also optimum in terms of algorithmic complexity, which guarantees the least execution time in practice.

\section{FURTHER DISCUSSIONS}

\subsection{Extensions of Proposed Approaches}

In Section 3, we focused on the distance-dominated power consumption and its role in topology control. Here, we relax these assumptions and reexamine our approaches in a more realistic context. For some particular WSNs, non-distancedominated or non-communication-related power consumptions may become nonnegligible (e.g., the power consumed within transmitter or receiver circuits or during data processing and view composition). Also, transmission power consumption may take a path loss exponent greater than 2, and include portions to combat multipath, shadowing, interference, and other air-link impairment. A third geometry dimension may be needed when node elevation varies considerably.

The approaches proposed in Section 3 are extensible to accommodate these changes. For example, we can still find the optimal BS location determined by a few critical ANs that run out of energy first. When we have the optimal $b_{N-1}$ for $N-1 \mathrm{ANs}$, for the $N$ th $\mathrm{AN}$, if its node lifetime with regard to $b_{N-1}$ is no less than the shortest one in these $N-1$ nodes, the $N$ th AN is noncritical, and $b_{N-1}$ is also optimal for this AN. Otherwise, the Nth AN is critical, and the recursive algorithm outlined in Table 3 still applies. Instead of Euclid distance used for a minimal enclosing circle or cone, the actual node lifetime should be used for comparison when determining whether an AN is logically enclosed from the prospective BS location. Line 7 in Table 3 can be extended to facilitate this. If node lifetime is not completely determined by its distance to the prospective BS location, we may no longer have a circle or cone to illustrate the process geometrically. However, the efficacy and optimality of the divide-and-conquer algorithms are still well-preserved. When the third geometry dimension is introduced, these approaches can also be extended into the third (or higher) dimension accordingly.

In addition to power consumption generalization for ANs, we may have some geographical constraints on the possible locations of BSs. Fig. 14 illustrates a case when the optimal $b$, denoted by filled triangle, has a linear constraint, i.e., $b$ should be located along a given line on the plane (e.g., an avenue across several buildings). Although $\left\{v_{1}, v_{2}, v_{3}\right\}$ still determine an acute triangle, $v_{3}$ is no longer critical in this case. The dashed small triangle denotes the optimal BS location without such a linear location constraint. To accommodate location constraints of BSs, we should extend line 2 in Table 3 accordingly. Specifically, we will take these constraints into account when we locate $b$ for a $V_{N}$ with a small $N \leq 3$. It is clear that such an extension affects neither the efficacy nor the optimality of the divide-and-conquer algorithms. Therefore, the proposed approaches also apply to location generalization for BSs.

\subsection{Topology Control Process: A Big Picture}

Locating BSs optimally constitutes a critical step of the entire topology control process. Given a geographical coverage and a set of information sources, before locating BSs, topology control first needs to determine where to place SN/AN clusters and how to allocate initial energy per $\mathrm{SN} / \mathrm{AN}$. The most intuitive approach for this step is to collocate SN/AN clusters with information sources and to begin with unit energy provisioning per cluster. Before actually locating BSs, a quick assessment can determine whether the initial energy provisioning is feasible for a WSN to maintain its desired network lifetime. If the desired lifetime is even more than the upper bound of maximal $L_{N}$ topological lifetime, applications should negotiate for $L_{N}^{K}$ or ${ }^{m} L_{N}^{K}$ lifetime, demand better initial energy provisioning, consider inter-AN relaying, or request more BSs. Since $L_{N} \leq{ }^{m} L_{N}^{K} \leq L_{N}^{K}$, the desired network lifetime may be met under either $K-o f-N$ or $m-i n-K-o f-N$ lifetime by exploiting deployment redundancy. Certainly, better initial energy provisioning can prolong network lifetime proportionally. An alternative is to introduce inter-AN relaying, if it is applicable and feasible, or place additional dedicated relay nodes (RNs) that can extend the lifetime of critical ANs. With more BSs, we effectively reduce $D$, which, in turn, increases achievable network lifetime. We did not address the problem of multiple BSs in this paper explicitly; however, since any AN should only associate with one BS, the proposed approaches still apply with regard to the chosen BS. On the other hand, if the desired network lifetime is less than the lower bound of $L_{N}$, we can simply locate the BS at the middle point of any two diameter ANs.

Another important step in topology control is to arrange inter-AN communications properly, if such activities are application applicable and energy favorable. In general, simultaneously locating the BS and arranging inter-AN relaying optimally to maximize network lifetime impose an NP-hard problem. A practical approach is to obtain the optimal BS location first and then arrange inter-AN relaying with regard to the located BS. If the BS location is known, we can obtain the optimal inter-AN relaying arrangement by designing a constrained network flow optimization problem. This problem can be solved by applying linear programming techniques [1]. Since normally ANs are equipped with at most one transmitter/receiver pair, it is critically important to design a serialized relay schedule, so that any AN always only needs to tune into a specific frequency band, time slot, or code sequence, i.e., communicating with at most one relaying $\mathrm{AN}$ at any given time. Although we did not address this step in this paper explicitly, locating the BS is a prerequisite step to make such an inter-AN relay arrangement possible. In practice, $\mathrm{SN} /$ ANs may fail and mission might be extended during its course; therefore, the topology control process needs to be revisited throughout the mission. In addition, a BS (e.g., a vehicle-mounted BS for a WSN) may be able to change its location when some ANs are about to run out of energy. This is very important for $L_{N}^{K}$ and ${ }^{m} L_{N}^{K}$ lifetimes, since ANs 
under these definitions may have various and dynamic priorities, and the BS should be able to accommodate them. When inter-AN relaying is available, the BS can relocate itself along the direction which further prolongs network lifetime heuristically.

\section{Related Work}

Mobile ad hoc networks (MANETs) and WSNs have attracted extensive research efforts in recent years. A comprehensive survey on WSNs can be found in [18] and the references therein. The research challenges and directions for MANETs can be found in [2]. Although sharing many similarities with MANET/WSNs, two-tiered WSNs have their unique underlying structures and application scenarios such as mission-driven AN/BS placement. Compared with MANET/WSNs, and despite their potential applications, two-tiered WSNs have not yet been adequately explored in the literature. In this section, we take both MANETs and regular WSNs as the references to review related work.

In MANETs, user mobility, network connectivity, and node reachability are major concerns. From the perspective of network protocols, source or intermediate nodes need to know how to route packets toward their destinations. Many unicast and multicast routing protocols are developed and evaluated [19], [8], [20], [9]. Multihop wireless communication in MANETs requests a revisit in radio transceiver, media access [4], [7], [5], and error or flow controls in link [21] or higher layers [22]. Many existing wireless MAC protocols, e.g., IEEE $802.11 \mathrm{a} / \mathrm{b} / \mathrm{g}$ and 802.15.1, were originally designed for wireless LAN/PANs; their applicability and achievable performance in MANETs and WSNs have been investigated [5]. Since user mobility plays an important role in MANETs, mobility management [23], location service [24], and their impact on routing and MAC protocols [25] have been studied as well.

WSNs may have a hybrid wireless multihop and BScentric infrastructure; they are more challenged by the limited on-board energy for disposable sensor and application nodes. For the multihop aspect, they share many similarities with MANETs in routing [26] and MAC protocols [6], along with additional energy constraints [27]. Unlike the user autonomy and flow independence properties of MANETs, information collected in WSNs has to be aggregated somewhere to magnify its value. Therefore, information flows from data sources, such as sensor or surveillance nodes, are likely to be structured (i.e., in a many-to-one manner) toward sinks, such as application nodes, cluster heads, or BSs, although the underlying forward paths may or may not be fixed. In addition, information collected by redundant and substitutable sources in dense WSNs may have considerable redundancy and inconsistency, so data fusion [28], [10] along the path or at the sink is very attractive. Application-specific fusion enables more sophisticated data [12] and node management functionalities inside WSNs, and can reduce unnecessary communication overhead significantly. Due to flow concentration, scalability can become an obstacle [29] for a large number of sensor nodes, and the techniques to introduce heterogeneity [30], hierarchy [31], clustering [11], [12], localization [32], and location-awareness [33] have been developed as well. These application characteristics add new dimensions to explore for WSNs in terms of MAC and multihop routing, and there are many results reported in the recent literature.
On the other hand, most research activities in WSNs assume a dense and microsensor deployment. Microsensors have very limited energy provisioning to capture scalaronly data such as temperature and motion triggered by external events. But, for a two-tiered WSN, ANs are much more capable than the ordinary microsensors (SNs), as they are required to construct and feed live local-views for BSs when they are activated. With the considerable coverage of a single SN/AN cluster, there is no need to have a very dense deployment of SN/AN clusters (generally, SN/AN clusters are placed with the proximity of designated scenes). Due to this sparse deployment, the inter-AN distance is comparable to the dimension of WSN coverage, and scalability is manageable even with a few BSs and a certain number of ANs. Based on these facts, the lifetime of an $\mathrm{AN}$ is dominated by its distance-related communication power consumption. Topology control, which determines the distance from ANs to BSs and chooses relays according to inter-AN distance, plays a vital role in maximizing network lifetime.

There are a few lifetime and topology-focused research activities in the literature. Bhardwaj et al. [34] derived the lifetime upper bound of information harvest sensor networks that convey probabilistic data from a point, a line, or an area source; they also gave simulation-based evaluations to validate the tightness of the derived bound. In [35], Bhardwaj et al. further explicitly formulated the optimal role assignment as the maximal network flow problem, again in data harvest networks where the BS is presumably located already. In this paper, our goal is to determine the optimal BS location to maximize topological lifetime even when relaying is infeasible. In addition, three definitions of topological lifetime are proposed according to mission criticality. Instead of harvesting from probabilistic sources, when being activated, WSNs should consistently offer an insitu, real-time, and steady global-view of the entire coverage.

Other topology-related research in WSNs mainly focused on multihop routings. For example, [36] considered fixed topologies of $\{4,6,8\}$-neighbor on a 2-dimension plane and 6-neighbor in a 3-dimension space, and proposed a poweraware routing scheme to reduce the total, and even the pernode, power consumption. In this paper, we have considered an arbitrary node placement on a plane, without any geometrical constraints on node neighborhood. In practice, the location of $\mathrm{SN} / \mathrm{AN}$ clusters is determined by specific missions, not by topology control. Ramanathan and Rosales-Hain [37] considered creating a desired topology of connectivity and biconnectivity by adjusting the transmitter output power. It was also observed that a poor topology can only offer a small fraction of the achievable lifetime, but they mainly focused on multihop networks without any common sinks such as BSs in WSNs. Schurgers et al. [38] proposed a sparse topology and energy management (STEM) technique that aggressively puts nodes in sleep mode and only wakes them up when they are needed to forward data. It also explored the equivalence of nearby nodes for data forwarding. However, in our two-tiered WSNs, due to their application characteristics, once being activated, the already-sparsely-deployed ANs usually cannot be forced into sleep. Otherwise, the designated local-views are lost. Wattenhofer et al. [39] proposed a 


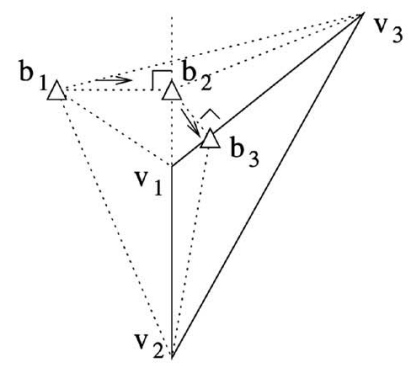

(a)

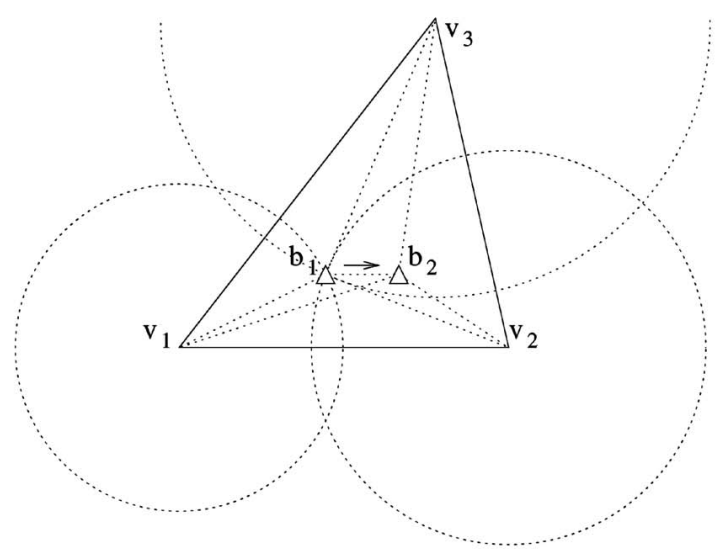

(b)

Fig. 15. $b$ should be inside $V_{3}$. (a) Moving $b$ toward $V_{3}$. (b) moving $b$ inside $V_{3}$.

distributed cone-based topological control to maintain the global connectivity with minimum power paths in multihop ad hoc networks; Chen et al. [40] considered a distributed algorithm to determine whether a node should be awake or asleep, depending on how many of its neighbors will get benefit and how much remaining energy the node has. The focus in these efforts, i.e., the purpose of topology control, is different from the one that we have in this paper. Instead of minimizing the power consumption of individual nodes or along a forwarding path, we minimize the power consumption of those ANs that dominate the network lifetime, or utility, of the entire WSN. Overall, the two-tiered WSNs considered in this paper are BS-centric with optional interAN relaying, where SN/AN clusters with a certain amount of initial energy are sparsely deployed in designated areas without significant redundancy.

\section{Conclusions}

In this paper, we have algorithmically obtained the optimal BS locations for two-tiered WSNs to maximize their topological lifetime under several definitions according to mission criticality. Most notably, we have obtained such locations even when the initial energy allocation of heterogeneous ANs is no longer proportional to their actual power consumption. In addition, we have analytically derived the upper and lower bounds of maximal topological lifetime by exploring some intrinsic properties of WSNs. Numerical evaluation has demonstrated the efficacy of topology control as a vital process for maximizing network lifetime of WSNs, and validated the optimality of the proposed approaches.

\section{APPENDIX A.1}

\section{Geometrical Properties of $\mathcal{C}$ When $V_{C}=V_{2}$}

As shown in Fig. 11, assume that $p=(x, y)$ satisfies $d_{p, v_{1}}: d_{p, v_{2}}=k_{1}: k_{2}$, where $v_{1}=\left(x_{1}, y_{1}\right)$ and $v_{2}=\left(x_{2}, y_{2}\right)$; i.e.,

$$
\sqrt{\left(x-x_{1}\right)^{2}+\left(y-y_{1}\right)^{2}}: \sqrt{\left(x-x_{2}\right)^{2}+\left(y-y_{2}\right)^{2}}=k_{1}: k_{2} .
$$

The property for $p$ can be rewritten as

$$
\begin{aligned}
k_{2}^{2}\left[\left(x-x_{1}\right)^{2}+\right. & \left.\left(y-y_{1}\right)^{2}\right]-k_{1}^{2}\left[\left(x-x_{2}\right)^{2}\right. \\
+ & \left.\left(y-y_{2}\right)^{2}\right]=0 \\
\left(k_{2}^{2}-k_{1}^{2}\right) x^{2}- & 2\left(k_{2}^{2} x_{1}-k_{1}^{2} x_{2}\right) x+\left(k_{2}^{2} x_{1}^{2}-k_{1}^{2} x_{2}^{2}\right) \\
+ & \left(k_{2}^{2}-k_{1}^{2}\right) y^{2}-2\left(k_{2}^{2} y_{1}-k_{1}^{2} y_{2}\right) x \\
+ & \left(k_{2}^{2} y_{1}^{2}-k_{1}^{2} y_{2}^{2}\right)=0 \\
& \left(x-\frac{k_{2}^{2} x_{1}-k_{1}^{2} x_{2}}{k_{2}^{2}-k_{1}^{2}}\right)^{2}+\left(y-\frac{k_{2}^{2} y_{1}-k_{1}^{2} y_{2}}{k_{2}^{2}-k_{1}^{2}}\right)^{2} \\
& \left(\frac{k_{1} k_{2} \sqrt{\left(x_{1}-x_{2}\right)^{2}+\left(y_{1}-y_{2}\right)^{2}}}{k_{1}^{2}-k_{2}^{2}}\right)^{2},
\end{aligned}
$$

i.e., all points having this property on the plane form a circle at

$$
c=\left(\frac{k_{2}^{2} x_{1}-k_{1}^{2} x_{2}}{k_{2}^{2}-k_{1}^{2}}, \frac{k_{2}^{2} y_{1}-k_{1}^{2} y_{2}}{k_{2}^{2}-k_{1}^{2}}\right)
$$

with radius $r=\frac{k_{1} k_{2} \sqrt{\left(x_{1}-x_{2}\right)^{2}+\left(y_{1}-y_{2}\right)^{2}}}{k_{1}^{2}-k_{2}^{2}}$. Since

$$
\begin{aligned}
d_{c, v_{1}}^{2} & =\frac{k_{1}^{2} k_{1}^{2}\left[\left(x_{1}-x_{2}\right)^{2}+\left(y_{1}-y_{2}\right)^{2}\right]}{\left(k_{2}^{2}-k_{1}^{2}\right)} \\
& <\frac{k_{1}^{2} k_{2}^{2}\left[\left(x_{1}-x_{2}\right)^{2}+\left(y_{1}-y_{2}\right)^{2}\right]}{\left(k_{2}^{2}-k_{1}^{2}\right)}=r^{2},
\end{aligned}
$$

$v_{1}$ is enclosed by this circle when $k_{1}<k_{2}$. Moreover, since

$$
\frac{\frac{k_{1}^{2}\left(y_{1}-y_{2}\right)}{k_{2}^{2}-k_{1}^{2}}}{\frac{k_{1}^{2}\left(x_{1}-x_{2}\right)}{k_{2}^{2}-k_{1}^{2}}}=\frac{y_{1}-y_{2}}{x_{1}-x_{2}},
$$

$c$ is collinear with $v_{1}$ and $v_{2}$, as shown in Fig. 11. When $k_{1}=k_{2}$, the equal-ratio points form a line. Geometrically, this line is equivalent to a circle centered at a point on line $v_{1} v_{2}$, with an infinite radius, and crossing line $v_{1} v_{2}$ at their midpoint.

It is worth pointing out that the above derivation is also reversible, i.e., if $p$ is on this circle $\left(d_{p, c}=r\right), \frac{d_{p, v_{1}}}{d_{p, v_{2}}}=\frac{k_{1}}{k_{2}}$. In addition, if $p$ is inside this circle or $d_{p, c}<r, \frac{d_{p, v_{1}}}{d_{p, v_{2}}}<\frac{k_{1}}{k_{2}}$; if $d_{p, c}>r, \frac{d_{p, v_{1}}}{d_{p, v_{2}}}>\frac{k_{1}}{k_{2}}$. 


\section{APPENDIX A.2}

\section{Geometrical Properties of $\mathcal{C}$ When $V_{C}=V_{3}$}

First, we show that $b$ should not be outside $V_{3}$; otherwise, we can always find another $p$ on one of the line segments determined by $V_{3}$, and $\max \left\{\frac{p v_{i}}{k_{i}}\right\}<\max \left\{\frac{b v_{i}}{k_{i}}\right\}$, which is a contradiction. As shown in Fig. 15a, the procedure is to project $b_{1}$ toward to the line determined by two nodes, e.g., $v_{1}$ and $v_{2}$, in $V_{3}$ that separate $b$ and the third node, $v_{3}$, in $V_{3}$. Due to the fact of projection, $d_{b_{1} v_{1}}>d_{b_{2} v_{1}}$ and $d_{b_{1} v_{2}}>d_{b_{2} v_{2}}$. Since $\angle b_{1} b_{2} v_{3}$ is an obtuse angle, $d_{b_{1} v_{3}}>d_{b_{2} v_{3}}$. Therefore, $\max \left\{\frac{b_{1} v_{i}}{k_{i}}\right\}>\max \left\{\frac{b_{2} v_{i}}{k_{i}}\right\}$. Similarly, $\max \left\{\frac{b_{2} v_{i}}{k_{i}}\right\}>\max \left\{\frac{b_{3} v_{i}}{k_{i}}\right\}$, and $b_{3}$ is on the line segment determined by $v_{1}$ and $v_{3}$. Through these projections, we can find that $b$ should not be outside $V_{C}$.

Next, we show that such a $b$ should be inside $V_{C}$ and at a location where $C_{1}$ and $C_{2}$ intersect at $b$, i.e., $\frac{d_{b v_{1}}}{k_{1}}=\frac{d_{b v_{2}}}{k_{2}}=\frac{d_{b v_{3}}}{k_{3}}$. Since it is impossible to find another $p$ on the plane that satisfies $d_{p v_{i}}<d_{b v_{i}}$ for $1 \leq i \leq 3$ simultaneously, $b$ is the optimal BS location that minimize $\max \left\{\frac{d_{p_{i}}}{k_{i}}\right\}$ for $1 \leq i \leq 3$. For example, as shown in Fig. 15b, $d_{b_{2} v_{2}}<d_{b_{1} v_{2}}$ and $d_{b_{2} v_{3}}<d_{b_{1} v_{3}}$, but $d_{b_{2} v_{1}}>d_{b_{1} v_{1}}$. Therefore, $\max \left\{\frac{d_{b_{2} v_{i}}}{k_{i}}\right\} \geq \frac{d_{b_{2} v_{1}}}{k_{1}}=$ $\max \left\{\frac{d_{b_{1} v_{i}}}{k_{i}}\right\}$ for $1 \leq i \leq 3$ when $b_{1}=b$, i.e., the optimal BS for heterogeneous ANs is at a location inside $V_{3}$ and where $C_{1}$ and $C_{2}$ intersect.

\section{ACKNOWLEDGMENTS}

This work has been supported in part by a Postgraduate Scholarship and a Strategic Research Grant from the Natural Science and Engineering Research Council (NSERC) of Canada, in part by US National Science Foundation (NSF) ANI-0312655, US Office of Naval Research (ONR) N00014-03-1-0521, and the Woodrow W. Everett, Jr. SCEEE Development Fund, USA. The authors also want to thank Dr. John Heidemann and the anonymous reviewers for their helpful comments on an early version of this paper. Preliminary results of this paper have been presented in part at ACM Mobicom '03 in San Diego [1].

\section{REFERENCES}

[1] J. Pan, Y. Hou, L. Cai, Y. Shi, and X. Shen, "Topology Control for Wireless Sensor Networks," Proc. of Ninth ACM Mobicom, pp. 286299, 2003.

[2] R. Ramanathan and J. Redi, "A Brief Overview of Ad Hoc Networks: Challenges and Directions," IEEE Comm. Magazine, vol. 40, no. 5, pp. 20-22, 2002.

[3] IEEE J. Selected Areas in Comm., special issue on wireless ad hoc networks, Z. Haas et al., eds., vol. 17, no. 8, 1999.

[4] E. Shih, S. Cho, N. Ickes, R. Min, A. Sinha, A. Wang, and A. Chandrakasan, "Physical Layer Driven Protocol and Algorithm Design for Energy-Efficient Wireless Sensor Networks," Proc. Seventh ACM Mobicom, pp. 272-287, 2001.

[5] A. Woo and D. Culler, "A Transmission Control Scheme for Media Access in Sensor Networks," Proc. of Seventh ACM Mobicom, pp. 221-235, 2001.

[6] W. Ye, J. Heidemann, and D. Estrin, "An Energy-Efficient MAC Protocol for Wireless Sensor Networks," Proc. 21st IEEE Infocom, pp. 1567-1576, 2002.

[7] E. Jung and N. Vaidya, "An Energy Efficient MAC Protocol for Wireless LANs," Proc. 21st IEEE Infocom, pp. 1756-1764, 2002.

[8] E. Royer and C-K. Toh, "A Review of Current Routing Protocols for Ad Hoc Wireless Networks," IEEE Personal Comm. Magazine, vol. 6, no. 2, pp. 46-55, 1999.
[9] S. Lee, W. Su, and M. Gerla, "Wireless Ad Hoc Multicast Routing with Mobility Prediction," Mobile Networks and Applications, vol. 6, no. 4, pp. 351-360, 2001.

[10] W. Heinzelman, J. Kulik, and H. Balakrishnan, "Adaptive Protocols for Information Dissemination in Wireless Sensor Networks," Proc. Fifth ACM Mobicom, pp. 174-185, 1999.

[11] A. Amis, R. Prakash, T. Vuong, and D. Huynh, "Max-Min DCluster Formation in Wireless Ad Hoc Networks," Proc. 19th IEEE Infocom, pp. 32-41, 2000.

[12] W. Heinzelman, A. Chandrakasan, and H. Balakrishnan, "EnergyEfficient Communication Protocols for Wireless Microsensor Networks," Proc. Hawaiian Int'l Conf. Systems Science, p. 8020, 2000.

[13] W. Heizelman, "Application-Specific Protocol Architecture for Wireless Networks," PhD thesis, MIT, 2000.

[14] J. Chou, D. Petrovis, and K. Ramchandran, "A Distributed and Adaptive Signal Processing Approach to Reducing Energy Consumption in Sensor Networks," Proc. 22nd IEEE Infocom, pp. 1054-1062, 2003.

[15] D. Niculescu and B. Nath, "Ad Hoc Positioning System (APS) Using AoA," Proc. 22nd IEEE Infocom, pp. 1734-1743, 2003.

[16] Y. Hou, Y. Shi, J. Pan, A. Efrat, and S. Midkiff, "Maximizing Lifetime of Wireless Sensor Networks through Single-Session Flow Routing," technical report, the Bradley Dept. of ECE, Virginia Tech, 2003.

[17] E. Welzl, "Smallest Enclosing Disks (Balls and Ellipsoids)," Lecture Notes in Computer Science, vol. 555, pp. 359-370, 1991.

[18] I. Akyildiz, W. Su, Y. Sankarasubramaniam, and E. Cayirci, "Wireless Sensor Networks: A Survey," Computer Networks, vol. 38, no. 4, pp. 393-422, 2002.

[19] S. Ramanathan and M. Steenstrup, "A Survey of Routing Techniques for Mobile Communications Networks," Mobile Networks and Applications, vol. 1, no. 2, pp. 89-104, 1996.

[20] J. Broch, D. Maltz, D. Johnson, Y. Hu, and J. Jetcheva, "A Performance Comparison of Multi-Hop Wireless Ad Hoc Network Routing Protocols," Proc. Fourth ACM Mobicom, pp. 85-97, 1998.

[21] M. Zorzi and R. Rao, "Error Control and Energy Consumption in Communications for Nomadic Computing," IEEE Trans. Computers, vol. 46, no. 3, 279-289, 1997.

[22] G. Holland and N. Vaidya, "Analysis of TCP Performance over Mobile Ad Hoc Networks," Wireless Networks, vol. 8, nos. 2-3, pp. 275-288, 2002.

[23] T. Camp, J. Boleng, and V. Davies, "A Survey of Mobility Models for Ad Hoc Network Research," Wireless Comm. \& Mobile Computing, vol. 2, no. 5, 483-502, 2002.

[24] J. Li, J. Jannotti, D. De Couto, D. Karger, and R. Morris, "A Scalable Location Service for Geographic Ad Hoc Routing," Proc. Sixth ACM Mobicom, pp. 120-130, 2000.

[25] C. Barrett, A. Marathe, M. Marathe, and M. Drozda, "Characterizing the Interaction between Routing and MAC Protocols in Ad Hoc Networks," Proc. Third ACM Mobihoc, pp. 92-103, 2002.

[26] J. Chang and L. Tassiulas, "Energy Conserving Routing in Wireless Ad Hoc Networks," Proc. 19th IEEE Infocom, pp. 22-31, 2000.

[27] C. Jones, K. Sivalingam, P. Agrawal, and J. Chen, "A Survey of Energy Efficient Network Protocols for Wireless Networks," Wireless Networks, vol. 7, no. 4, pp. 343-358, 2001.

[28] C. Intanagonwiwat, R. Govindan, and D. Estrin, "Directed Diffusion: A Scalable and Robust Communication Paradigm for Sensor Networks," Proc. Sixth ACM Mobicom, pp. 56-67, 2000.

[29] D. Estrin, R. Govindan, J. Heidemann, and S. Kumar, "Next Century Challenges: Scalable Coordination in Sensor Networks," Proc. Fifth ACM Mobicom, pp. 263-270, 1999.

[30] E. Duarte-Melo and M. Liu, "Analysis of Energy Consumption and Lifetime of Heterogeneous Wireless Sensor Networks," Proc. 45th IEEE Globecom, pp. 21-25, 2002.

[31] F. Ye, H. Luo, J. Cheng, S. Lu, and L. Zhang, "A Two-Tier Data Dissemination Model for Large Scale Wireless Sensor Networks," Proc. Seventh ACM Mobicom, pp. 148-159, 2001.

[32] K. Sohrabi, J. Gao, V. Ailawadhi, and G. Pottie, "Protocols for SelfOrganization of a Wireless Sensor Network," IEEE Personal Comm., vol. 7, no. 5, pp. 16-27, 2000.

[33] Y. Xu, J. Heidemann, and D. Estrin, "Geography-Informed Energy Conservation for Ad Hoc Routing," Proc. Seventh ACM Mobicom, pp. 70-84, 2001.

[34] M. Bhardwaj, A. Chandrakasan, and T. Garnett, "Upper Bounds on the Lifetime of Sensor Networks," Proc. 36th IEEE Int'l Conf. Comm. (ICC), pp. 785-790, 2001. 
[35] M. Bhardwaj and A. Chandrakasan, "Bounding the Lifetime of Sensor Networks via Optimal Role Assignment," Proc. 21st IEEE Infocom, pp. 1587-1596, 2002.

[36] A. Salhieh, J. Weinmann, M. Kochha, and L. Schwiebert, "Power Efficient Topologies for Wireless Sensor Networks," Proc. Int'l Conf. Parallel Processing, pp. 156-163, 2001.

[37] R. Ramanathan and R. Rosales-Hain, "Topology Control of Multihop Wireless Networks Using Transmit Power Adjustment," Proc. Ninth IEEE Infocom, pp. 404-413, 2000.

[38] C. Schurgers, V. Tsiatsis, S. Ganeriwal, and M. Srivastava, "Topology Management for Sensor Networks: Exploiting Latency and Density," Proc. Third ACM Mobihoc, pp. 135-145, 2002.

[39] R. Wattenhofer, L. Li, P. Bahl, and Y. Wang, "Distributed Topology Control for Wireless Multihop Ad Hoc Networks," Proc. 20th IEEE Infocom, pp. 1388-1397, 2001.

[40] B. Chen, K. Jamieson, H. Balakrishnan, and R. Morris, "SPAN: An Energy Efficient Coordination Algorithm for Topology Maintenance in Ad Hoc Wireless Networks," Proc. Seventh ACM Mobicom, pp. 85-96, 2001.

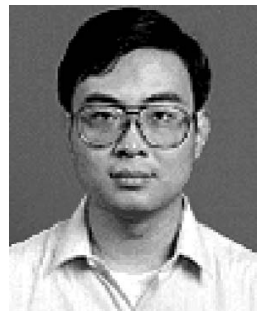

Jianping Pan (S'96-M'99) received the BS and $\mathrm{PhD}$ degrees in computer science from Southeast University, Nanjing, China, in 1994 and 1998, respectively. From 1999 to 2001, he was a postdoctoral fellow and then a research associate with the Centre for Wireless Communications at the University of Waterloo, Waterloo, ON, Canada. From 2001 to 2003, he was a member of research staff with Fujitsu Laboratories of America, Sunnyvale, California. Since 2003, he has been a research scientist at NTT MCL, Palo Alto, California. His research interests include protocols and applications for high-speed, multimedia, and mobile networks. He is a member of the IEEE, the IEEE Computer Society, and the ACM.

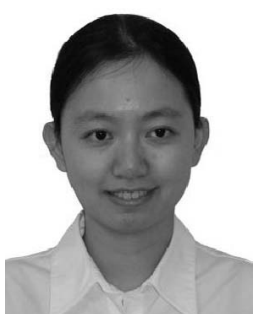

Lin Cai $\left(S^{\prime} 00\right)$ received the MASc degree in electrical and computer engineering from the University of Waterloo, Waterloo, Canada, in 2002. She is currently pursuing the $\mathrm{PhD}$ degree in the same field at the University of Waterloo. She received a postgraduate scholarship from the Natural Sciences and Engineering Research Council of Canada (NSERC) in 2003. Her research interests span several areas in wireless communications and networking, with a focus on network protocol and architecture design supporting emerging multimedia applications over IP-based wireless, mobile, ad hoc, and sensor networks. She is a student member of the IEEE.

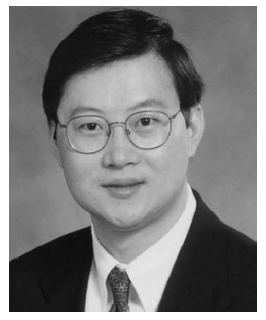

Y. Thomas Hou (S'91-M'98-SM'04) received the BE degree from the City College of New York in 1991, the MS degree from Columbia University in 1993, and the PhD degree from Polytechnic University, Brooklyn, New York, in 1998, all in electrical engineering. From 1997 to 2002, Dr. Hou was a research scientist and project leader at Fujitsu Laboratories of America, Networking Research Department, Sunnyvale, California (Silicon Valley). He joined Virginia Tech, The Bradley Department of Electrical and Computer Engineering, Blacksburg, Virginia, in the Fall of 2002 and is currently an assistant professor. Dr. Hou's research interests are in the algorithm design and optimization for network systems. His current research focuses on wireless sensor and ad hoc networks and multimedia over wireless ad hoc networks. In recent years, he has worked on scalable architectures, protocols, and implementations for differentiated services Internet; service overlay networking; multimedia streaming over the Internet; and network bandwidth allocation policies and distributed flow control algorithms. He has published extensively in the above areas and is a corecipient of the 2002 IEEE International Conference on Network Protocols (ICNP) Best Paper Award and the 2001 IEEE Transactions on Circuits and Systems for Video Technology (CSVT) Best Paper Award. $\mathrm{He}$ is a senior member of the IEEE and the IEEE Computer Society, and a member of the ACM.

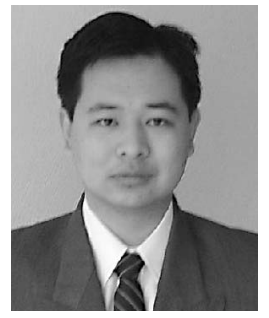

Yi Shi (S'03) received the BS degree from the University of Science and Technology of China, Hefei, China, in 1998, the MS degree from the Institute of Software, Chinese Academy of Science, Beijing, China, in 2001, and a second MS degree from Virginia Tech, Blacksburg, Virginia, in 2003, all in computer science. He is currently working toward the $\mathrm{PhD}$ degree in electrical and computer engineering at Virginia Tech. While an undergraduate, he was a recipient of the Meritorious Award in International Mathematical Contest in Modeling in 1997 and 1998, respectively. His current research focuses on algorithms and optimization for wireless sensor networks and wireless ad hoc networks. His work has appeared in highly selective international conferences (e.g., MobiCom and MobiHoc). He is a student member of the IEEE.

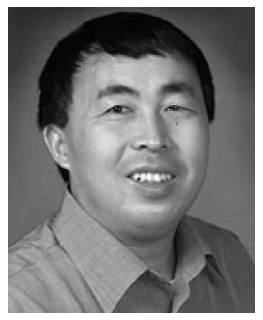

Sherman X. Shen (M'97-SM'02) received the BSc (1982) degree from Dalian Marine University, China, and the MSc (1987) and PhD degrees (1990) from Rutgers University, New Jersey, all in electrical engineering. From September 1990 to September 1993, he was first with the Howard University, Washington D.C., and then the University of Alberta, Edmonton (Canada). Since October 1993, he has been with the Department of Electrical and Computer Engineering, University of Waterloo, Canada, where he is a full professor. Dr. Shen's research focuses on mobility and resource management in interconnected wireless/wireline networks, UWB wireless communications, ad hoc networks, stochastic process, and control. $\mathrm{He}$ is a coauthor of three books and has publications in communications networks, control, and filtering. Dr. Shen serves as the technical cochair for the IEEE Globecom'03 Symposium on Next Generation Networks and Internet, and International Symposium on Parallel Architectures, Algorithms, and Networks, 2004; the editor for the IEEE Transactions on Wireless Communications and Dynamics of Continuous, Discrete and Impulse Systems, Series B: Application and Algorithm —an International Journal; the associate editor for Wireless Communications and Mobile Computing (John Wiley) and the International Journal of Computers and Applications, and guest editor for the IEEE Journal of Selected Areas in Communications, special issue on ultra wideband wireless communications, the IEEE Communications Magazine, special issue on China wireless communications-technology versus markets, and IEEE Wireless Communications, special issue on $4 \mathrm{G}$ mobile communicationtowards open wireless architecture. Dr. Shen received the Premier's Research Excellence Award (PREA) from the Province of Ontario for demonstrated excellence of scientific and academic contributions in 2003, and the Distinguished Performance Award from the Faculty of Engineering, University of Waterloo, for outstanding contribution in teaching, scholarship and service in 2002. Dr. Shen is a senior member of the IEEE and the IEEE Computer Society, and a registered Professional Engineer of Ontario, Canada.

$\triangleright$ For more information on this or any other computing topic, please visit our Digital Library at www.computer.org/publications/dlib. 